\title{
Numerical simulations of expanding supershells in dwarf irregular galaxies
}

\section{Formation of giant $\mathrm{HI}$ rings}

\author{
E. I. Vorobyov ${ }^{1,2}$ and Shantanu Basu ${ }^{2}$ \\ ${ }^{1}$ Institute of Physics, Stachki 194, Rostov-on-Don, Russia \\ e-mail: eduard_vorobev@mail.ru \\ 2 Department of Physics and Astronomy, University of Western Ontario, London, Ontario N6A 3K7, Canada \\ e-mail: basu@astro.uwo.ca
}

Received 19 May 2004 / Accepted 14 October 2004

\begin{abstract}
We perform numerical hydrodynamic modeling of various physical processes that can form an $\mathrm{HI}$ ring as is observed in Holmberg I (Ho I). Three energetic mechanisms are considered: multiple supernova explosions (SNe), a hypernova explosion associated with a gamma ray burst (GRB), and the vertical impact of a high velocity cloud (HVC). The total released energy has an upper limit of $\sim 10^{54} \mathrm{erg}$. We find that multiple SNe are in general more effective in producing shells that break out of the disk than a hypernova explosion of the same total energy. As a consequence, multiple SNe form rings with a high ringto-center contrast $\mathcal{K} \lesssim 100$ in the HI column density, whereas single hypernova explosions form rings with $\mathcal{K} \lesssim 10$. Only multiple SNe can reproduce both the size (diameter $\sim 1.7 \mathrm{kpc}$ ) and the ring-to-center contrast $(\mathcal{K} \sim 15-20)$ of the HI ring in Ho I. High velocity clouds create HI rings that are much smaller in size $(\lessgtr 0.8 \mathrm{kpc})$ and contrast $(\mathcal{K} \lesssim 4.5)$ than seen in Ho I. We construct model position-velocity $(\mathrm{pV})$ diagrams and find that they can be used to distinguish among different HI ring formation mechanisms. The observed pV-diagrams of Ho I (Ott et al. 2001) are best reproduced by multiple SNe. We conclude that the giant $\mathrm{HI}$ ring in Ho I is most probably formed by multiple SNe. We also find that the appearance of the SNe-driven shell in the integrated $\mathrm{HI}$ image depends on the inclination angle of the galaxy. In nearly face-on galaxies, the integrated $\mathrm{HI}$ image shows a ring of roughly constant HI column density surrounding a deep central depression, whereas in considerably inclined galaxies $\left(i>45^{\circ}\right)$ the $\mathrm{HI}$ image is characterized by two kidney-shaped density enhancements and a mild central depression.
\end{abstract}

Key words. galaxies: dwarf - ISM: bubbles

\section{Introduction}

There are a few nearby low-mass dwarf irregular galaxies (dIrr's) such as Holmberg I (Tully et al. 1978; Ott et al. 2001), M 81 dwA (Sargent et al. 1983; Westpfahl \& Puche 1993), Sagittarius DIG (Young \& Lo 1997), Sextans A (Skillman et al. 1988; Stewart 1998), the HI morphology of which is totally dominated by a single ring structure of size comparable to or bigger than their optical extent. The integrated HI image of these dIrr's shows a central HI hole surrounded by a denser $\mathrm{HI}$ ring. The contrast in the azimuthally averaged $\mathrm{HI}$ column density between the central hole and the ring varies from $\sim 3-4$ in Sextans A and M 81 dwA to $~ 15-20$ in Holmberg I. HI rings that do not dominate the overall HI extent of a galaxy, yet occupy a considerable region (kpc scale), have also been found in other dIrr's such as DDO 47 (shell 13, according to Walter \& Brinks 2001) and Holmberg II (shell 21, according to Puche et al. 1992).

There are a number of scenarios that could create HI holes in galactic disks (see Sánchez-Salcedo 2002), which can loosely be divided into two groups comprising the energetic and non-energetic mechanisms. The energetic mechanisms assume a deposition of vast amount of energy into the interstellar medium and include multiple supernova explosions (Mac Low \& McCray 1988; De Young \& Heckman 1994; Mac Low \& Ferrara 1999; Silich et al. 2001), impact of high velocity clouds (Tenorio-Tagle et al. 1987; Comerón \& Torra 1992; Rand \& Stone 1996), and gamma ray bursts (Efremov et al. 1998; Loeb \& Perna 1998). The non-energetic mechanisms include a combined action of thermal and gravitational instabilities in the gas disk (Wada et al. 2000), turbulent clearing (Elmegreen 1997; Walter \& Brinks 1999), and ultra-violet erosion of the HI disk (Vorobyov \& Shchekinov 2004). It appears however very unlikely that a central HI depression surrounded by a denser $\mathrm{HI}$ ring with size comparable to the galaxy's optical extent could be produced by a non-energetic mechanism. Indeed, Vorobyov et al. (2004) have recently shown that the HI ring-like morphology of Holmberg I (Ho I) can be produced by multiple supernova explosions ( $\mathrm{SNe})$. 
In this paper, we consider two other energetic mechanisms of HI ring formation, namely the impact of high velocity clouds (HVCs) and gamma ray bursts (GRBs), and show that they cannot explain the formation of the observed giant $\mathrm{HI}$ ring in Ho I. We generate the position-velocity diagrams and show that they can be used to distinguish between the rings created by different energetic mechanisms. We find that the appearance of $\mathrm{HI}$ rings created by multiple $\mathrm{SNe}$ is sensitive to the inclination angle of the galaxy. In considerably inclined galaxies, the HI ring would rather appear as two kidney-shaped density enhancements similar to those observed in Sectans A.

The paper is organized as follows. In Sect. 2 the numerical hydrodynamic model for simulating multiple SNe, GRBs, and the impact of HVCs is formulated. A comparative study of multiple SNe and GRBs of the same total energy is performed in Sect. 4. The collision of HVCs with the galactic gas disk is considered in Sect. 5. The main results are summarized in Sect. 7.

\section{Numerical model}

Our model galaxy consists of a rotating gas disk, stellar disk, and a spherically symmetric dark matter halo. The density profile of the stellar component is chosen as:

$\rho=\rho_{\mathrm{s} 0} \operatorname{sech}^{2}\left(z / z_{\mathrm{s}}\right) \exp \left(-r / r_{\mathrm{s}}\right)$,

where $\rho_{\mathrm{s} 0}$ is the stellar density in the center of the galaxy, and $z_{\mathrm{s}}$ and $r_{\mathrm{s}}$ are the vertical scale height and radial scale length of the stellar component, respectively. We assume that the density profile of the dark matter (DM) halo can be approximated by a modified isothermal sphere (Binney \& Tremaine 1987)

$\rho_{\mathrm{h}}=\frac{\rho_{\mathrm{h} 0}}{1+\left(r / r_{\mathrm{h}}\right)^{2}}$,

where the central density $\rho_{\mathrm{h} 0}$ and the characteristic scale length $r_{\mathrm{h}}$ were given by Mac Low \& Ferrara (1999) and Silich $\&$ Tenorio-Tagle (2001) based on the study of the dark-tovisible mass ratios by Persic et al. (1996):

$\rho_{\mathrm{h} 0}=6.3 \times 10^{10}\left(\frac{M_{\mathrm{h}}}{M_{\odot}}\right)^{-1 / 3} h^{-1 / 3} M_{\odot} \mathrm{kpc}^{-3}$,

$r_{\mathrm{h}}=0.89 \times 10^{-5}\left(\frac{M_{\mathrm{h}}}{M_{\odot}}\right)^{1 / 2} h^{1 / 2} \mathrm{kpc}$.

Here, $h$ is the Hubble constant in units of $100 \mathrm{~km} \mathrm{~s}^{-1} \mathrm{Mpc}^{-1}$ and $M_{\mathrm{h}}$ is the total halo mass. We adopt $h=0.65$ throughout the paper.

Our model galaxy is meant to represent Ho I and we use the observations of Ott et al. (2001) to constrain the parameters of our model. We note that $M_{\mathrm{h}}$ in Eqs. (3) and (4) is, in fact, the total mass of the halo. We vary $M_{\mathrm{h}}$ until the actual halo mass confined within the HI diameter of Ho I $(5.8 \mathrm{kpc}$, Ott et al. 2001) agrees with the observed value, $3.1 \times 10^{8} M_{\odot}$. This fit yields a value of $M_{\mathrm{h}}=6.0 \times 10^{9} M_{\odot}$ for Ho I. Once the total halo mass is fixed, we derive the parameters of the halo density distribution using Eqs. (3) and (4), which are further used to compute the gravitational potential of the halo as described in

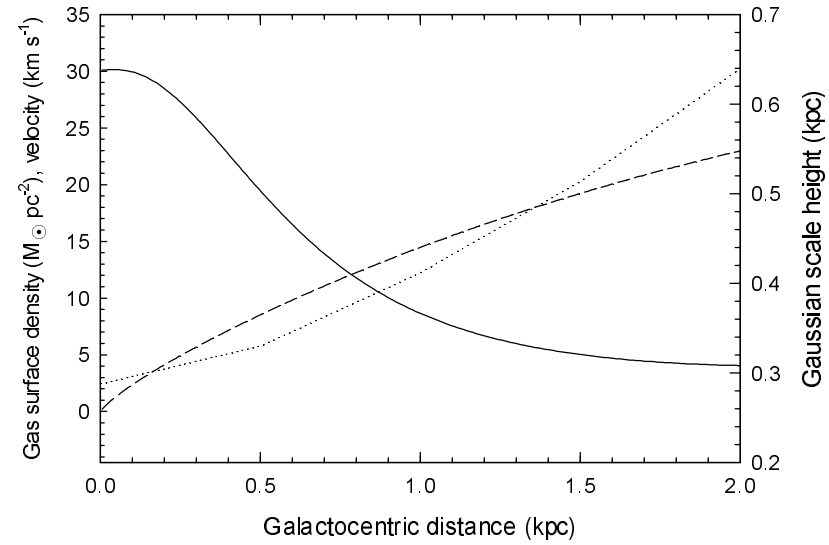

Fig. 1. The equilibrium radial distribution of gas surface density (the solid line), the initial rotation curve (the dashed line), and the Gaussian vertical scale height of gas distribution (the dotted line).

Vorobyov et al. (2004). We have adopted a value of $z_{\mathrm{s}}=300 \mathrm{pc}$, which is typical for dwarf irregular galaxies. The radial scale length $r_{\mathrm{s}}=1.7 \mathrm{kpc}$ of the stellar disk is estimated from the $I_{\mathrm{c}}-$ band radial surface brightness profile of Ho I. With $r_{\mathrm{s}}$ and $z_{\mathrm{s}}$ being fixed, the stellar density $\rho_{\mathrm{s} 0}$ in Eq. (1) is varied so as to obtain the measured luminous stellar mass of $M_{\mathrm{s}}=1.0 \times 10^{8} M_{\odot}$. This results in $\rho_{\mathrm{s} 0} \approx 0.02 M_{\odot} \mathrm{pc}^{-3}$. The adopted parameters of the stellar disk are further used to compute the stellar gravitational potential by solving the Poisson equation.

Once the stellar and DM halo gravitational potentials are fixed, we obtain the initial gas density distribution by solving the steady-state momentum equation as described in Vorobyov et al. (2004). We set the gas velocity dispersion $\sigma=(R T / \mu)^{1 / 2}$ to be $9 \mathrm{~km} \mathrm{~s}^{-1}$ (Ott et al. 2001). We vary the rotation curve until the initial gas surface density distribution becomes exponential, which is in agreement with observations of the HI radial distribution in many dIrr's (Taylor et al. 1994). The observed rotation curve (RC) of Ho I is known to the accuracy of the inclination angle, which in turn is poorly determined (Ott et al. 2001) due to Ho I's small inclination. We have chosen the initial RC in our model so that the initial radial gas surface density distribution well reproduces the observed profile at $r>1 \mathrm{kpc}$, i.e. at radii which are not affected by the subsequent ring expansion. We do not expect our initial RC to match the currently observed RC, since the latter is already affected by ring formation. Furthermore, accurately modeling the observed RC (for an assumed inclination angle) requires nonaxisymmetric simulations, since the dynamical center of Ho I is $\sim 0.7 \mathrm{kpc}$ offset from the morphological center of the ring. This offset may complicate the shape of the observed RC, because it is measured around the dynamical center. This task is beyond the scope of our paper. The resulting radial gas surface density profile, as well as the Gaussian vertical scale height $h$ of the gas distribution and initial gas rotation curve, are plotted in Fig. 1 by the solid, dotted, and dashed lines, respectively. The total gas mass within the computational domain is $M_{\text {gas }}=1.0 \times 10^{8} M_{\odot}$, of which $\sim 30 \%$ is contributed by $\mathrm{He}$ $\left(M_{\mathrm{HI}+\mathrm{He}} \approx 1.4 M_{\mathrm{HI}}, \mu=1.27\right.$, Brinks 1990). This value of $M_{\mathrm{gas}}$ roughly agrees with that obtained by Ott et al. (2001). 
A usual set of hydrodynamical equations in cylindrical coordinates (with the assumption of axial symmetry) is solved using the method of finite-differences with a time-explicit, operator-split solution procedure as used in the ZEUS-2D code described in detail in Stone \& Norman (1992). The computational domain spans the range of $7.2 \mathrm{kpc} \times 2 \mathrm{kpc}$ in the vertical and horizontal directions, respectively, with a resolution of $5 \mathrm{pc}$. We have implemented the optically thin cooling curve given in Wada \& Norman (2001) for a metallicity of one tenth of solar, which is typical for dIrr's. The cooling processes taken into account are: (1) recombination of $\mathrm{H}, \mathrm{He}, \mathrm{C}, \mathrm{O}, \mathrm{N}, \mathrm{Si}$, and Fe; (2) collisional excitation of HI, CI-IV, and OI-IV; (3) hydrogen and helium bremsstrahlung; (4) vibrational and rotational excitation of $\mathrm{H}_{2}$; (5) atomic and molecular cooling due to fine-structure emission of $\mathrm{C}, \mathrm{C}+$, and $\mathrm{O}$, and rotational line emission of $\mathrm{CO}$ and $\mathrm{H}_{2}$. We use an empirical heating function tuned to balance the cooling in the background atmosphere so that it maintains the gas in hydrostatic and thermal equilibrium; it may be thought of as a crude model for the stellar energy input. However, heating is prohibited at $T_{\text {gas }}>2.0 \times 10^{4} \mathrm{~K}$ to avoid the effects of spurious heating of a bubble's interior by the time-independent heating function. This is physically justified since most of the heating in the warm interstellar medium comes from the photoelectric heating of polycyclic aromatic hydrocarbon molecules (PAHs) and small grains, which will be either evaporated or highly ionized in the bubble filled with hot supernova ejecta. Cooling and heating are treated numerically at the end of the time integration step using an implicit update to the energy equation. The implicit equation for energy density is solved by Newton-Raphson iteration, supplemented by a bisection algorithm for occasional zones where the NewtonRaphson method does not converge. In order to monitor accuracy, the total change in the internal energy density in one time step is kept below $15 \%$. If this condition is not met, the time step is reduced and a solution is again sought. In the following we give a brief description on the mechanisms of energy injection used in our numerical simulation.

\section{Energetic mechanisms of $\mathrm{HI}$ ring formation}

Here, we briefly review possible mechanisms of HI ring formation as observed in Ho I.

Consecutive supernova explosions. The origin of the giant $\mathrm{HI}$ rings in dIrr's is traditionally thought to lie in the combined effect of stellar winds and supernova explosions produced by young stellar associations (see e.g. Ott et al. 2001). In our simulations, the energy of supernova explosions is released in the form of thermal energy in the central region with a radius of $30 \mathrm{pc}$ filled with the hot $\left(T \sim 10^{7} \mathrm{~K}\right)$ and rarified $\left(n \sim 10^{-3} \mathrm{~cm}^{-3}\right)$ gas, which is presumably formed by the previous action of stellar winds. We use a constant wind approximation, i.e. at each time step we add energy to the source region at a rate of $\dot{E}=L$, where $L$ is the mechanical luminosity defined as the total released energy of SNe divided by the duration of the energy input phase. We choose the energy input phase to last for $30 \mathrm{Myr}$, which corresponds roughly to the difference in the lifetimes of the most and least massive stars capable of producing $\mathrm{SNe}$ in a cluster of simultaneously born stars. Since in the present simulations we deal with large stellar clusters with hundreds of supernovae, the release of the energy of $\mathrm{SNe}$ in the form of thermal energy is justified (Mac Low \& McCray 1988).

Gamma ray bursts. The consecutive SN explosions may not be the only mechanism that could release $10^{53}-10^{54} \mathrm{erg}$ of energy, enough to form giant HI rings. Another mechanism has been suggested by Efremov et al. (1998) and Loeb \& Perna (1998), who argued that the GRB explosions are powerful enough to make kpc-size shells in the interstellar media of spiral and irregular galaxies. Although the physics of GRBs is still poorly understood, the general picture emerging is that they are highly energetic events $\left(\leq 10^{54} \mathrm{erg}\right.$, see e.g. Paczyński 1998) that release energy in a short period of time (of the order of a few seconds). Consequently, we model the GRB explosion (hereafter, "hypernova" according to Paczyński 1998) by an instantaneous release of thermal energy within a sphere of $30 \mathrm{pc}$, filled with hot $\left(T \sim 10^{7} \mathrm{~K}\right)$ and rarified $\left(n \sim 10^{-3} \mathrm{~cm}^{-3}\right)$ gas. We have also explored the injection of hypernova energy in the form of kinetic energy and found that it does not noticeably influence the dynamics of the hypernova-driven shell.

Impact of high velocity clouds. Another possible mechanism that could create HI holes in the galactic disks was proposed by Tenorio-Tagle et al. (1987), who argued that the infall of high velocity clouds could deposit $10^{52}$ to $10^{54}$ erg per collision. The numerical simulations of Tenorio-Tagle et al. (1987) have indeed shown that HVCs are capable of forming the giant curved arcs and cavities in the Galactic disk (see also Rand \& Stone (1996) for numerical simulations of HVC impact in NGC 4631). Most previous numerical simulations have been concerned with the infall of HVCs in massive galaxies, because their collisional cross section is much larger than that of dwarf irregulars. Moreover, the gas disks in dwarf irregulars are in general thicker than those of massive spiral galaxies, which would make it more difficult for an HVC to penetrate the disk. In summary, the formation of a giant $\mathrm{HI}$ hole surrounded by a denser HI ring (as is observed in Ho I and other dwarf irregulars) by the infall of HVCs is not obvious and requires further investigation.

Taking into account the diameter of the HI ring in Ho I $(\sim 1.7 \mathrm{kpc})$, we consider the most energetic HVCs, which cover a velocity range $200 \mathrm{~km} \mathrm{~s}^{-1} \leq v_{\mathrm{HVC}} \leq 300 \mathrm{~km} \mathrm{~s}^{-1}$ and have $\mathrm{HI}$ column density $10^{20} \mathrm{~cm}^{-3} \leq N_{\mathrm{HI}} \leq 10^{21} \mathrm{~cm}^{-3}$. In our simulations the kinetic energies of HVCs range from $1.0 \times 10^{53}$ to $2.5 \times 10^{54} \mathrm{erg}$, which corresponds to a variation in HVC masses of $2.5 \times 10^{5} M_{\odot}$ to $2.5 \times 10^{6} M_{\odot}$. Such massive and energetic HVCs most probably have an extragalactic origin.

\section{Supernova explosions versus gamma ray bursts}

We start by showing in Fig. 2 the temporal evolution of the distribution of the gas volume density produced by 200 consecutive $\mathrm{SNe}$ (the upper panels) and by a hypernova explosion of the same total energy of $2 \times 10^{53} \mathrm{erg}$ (the lower panels). The overall gas dynamics is similar in both cases. However, there are minor differences seen not only in the dynamics of the hot gas but also in the shape of the blown-up shell. In the case of a hypernova explosion, there is no "blowout" observed (i.e. the 


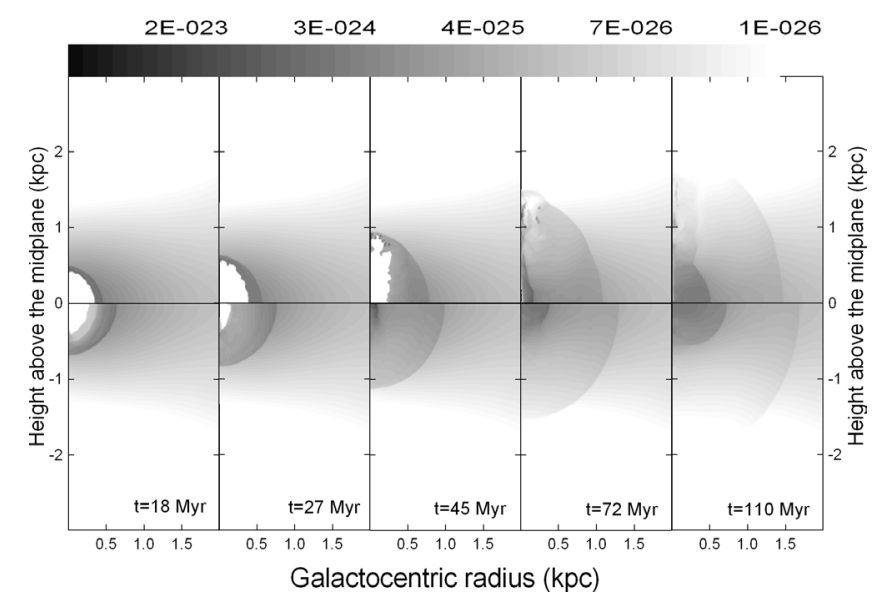

Fig. 2. Temporal evolution of the gas volume density distribution after the release of $2 \times 10^{53} \mathrm{erg}$ of thermal energy at $z=0, r=0 \mathrm{pc}$. The upper panels correspond to 200 consecutive SN explosions over $30 \mathrm{Myr}$, while the lower panels show the impact of a single hypernova of the same total energy of $2.0 \times 10^{53} \mathrm{erg}$. The scale bar is in $\mathrm{gm} \mathrm{cm}^{-3}$.

shell breaking out of the disk and pumping the hot gas into the intergalactic medium). The hot gas is always confined inside the shell in the early expansion phase and it cools down at later times $t>40$ Myr due to the radiative cooling. As a result, the shell collapses at $t=45 \mathrm{Myr}$, creating a mildly compressed central core, the gas density of which is however below the Jeans limit. In the case of multiple SNe, part of the hot gas is lifted to a higher altitude of $z \geq 1 \mathrm{kpc}$ due to a buoyancy effect developing at $t \approx 45 \mathrm{Myr}$. It is also seen that in the early expansion phase the shell is on average thicker in the case of the hypernova explosion than in the case of multiple SN explosions. Since the numerical modeling of Vorobyov et al. (2004) suggested that the shell in Ho I has already blown out of the disk, we do not consider further the energy release of $2.0 \times 10^{53} \mathrm{erg}$.

The difference in the shell dynamics between the multiple $\mathrm{SN}$ and hypernova becomes pronounced as one considers more energetic explosions. For instance, Fig. 3 shows the distribution of the gas volume density produced by 500 consecutive SN explosions (the upper panels) and by a single hypernova explosion of the same total energy $5.0 \times 10^{53}$ erg (the lower panels). Now, the dynamics of the $\mathrm{SN}$-driven shell shows a clear blowout phase at $t \approx 45 \mathrm{Myr}$ after the beginning of the energy input phase. On the contrary, the hypernova-driven shell never breaks out of the disk, though its energy is equal to the total energy released by multiple supernovae. This difference remains for presumably an upper limit energy release of $10^{54}$ erg that a stellar cluster could produce in Holmberg I. As seen in the upper panels of Fig. 4, the shell dynamics governed by 1000 consecutive SN explosions exhibits a violent blowout, whereas that governed by a hypernova of the same total energy of $10^{54} \mathrm{erg}$ shows almost no sign of blowout. This is in good agreement with the previous model of Efremov et al. (1999), who used a thin shell approximation to study the evolution of shells formed by a single hypernova and multiple SNe. These authors have also found that an abrupt energy input creates shells that do not blow out to the galactic halo for energies $\lesssim 10^{54} \mathrm{erg}$. Noticeably, the final fate of the gas distribution

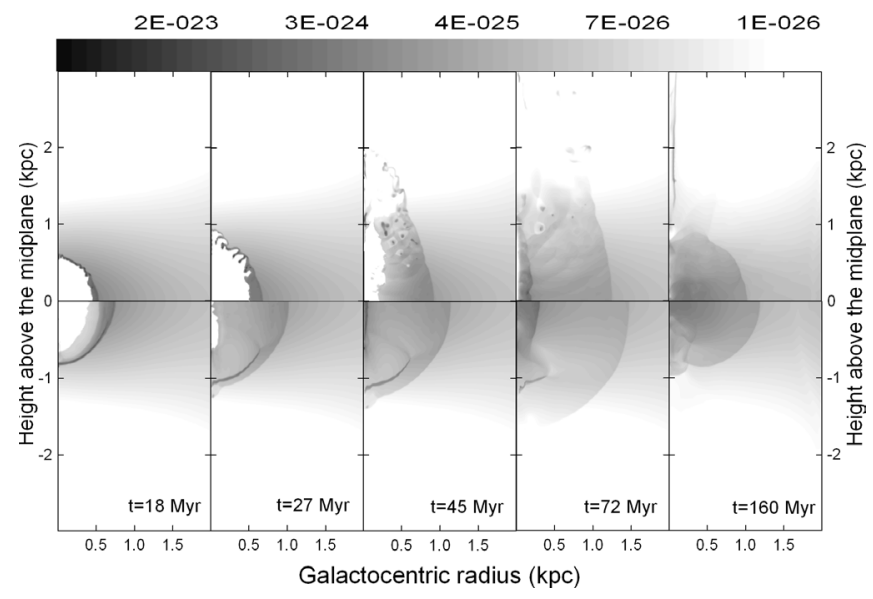

Fig. 3. The same as Fig. 2, but for the energy input of $5 \times 10^{53} \mathrm{erg}$.

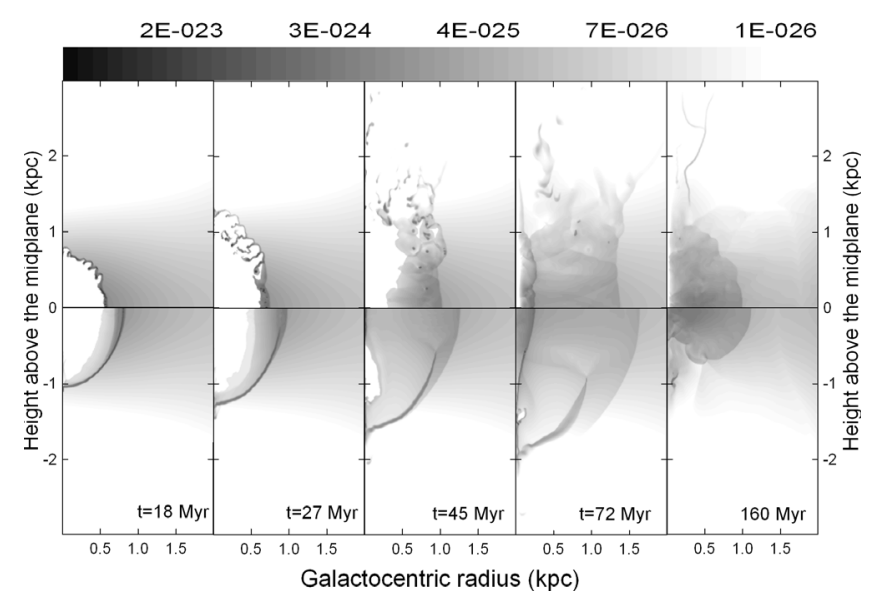

Fig. 4. The same as Fig. 2, but for the energy input of $10^{54} \mathrm{erg}$.

is similar in all cases: the hot bubble fills in and the gas disk mostly recovers its pre-explosion appearance after $\geq 160 \mathrm{Myr}$ with a slightly more centrally condensed radial gas distribution.

The occurrence of the blowout in the case of multiple supernova explosions and its absence in the case of a hypernova explosion can be understood if one considers the dynamics of the hot gas filling the shell interior. In Fig. 5 we plot the gas volume density distribution and velocity field produced by 500 consecutive SN explosions (the left panel) and a hypernova of the same total energy of $5.0 \times 10^{53} \mathrm{erg}$ (the right panel). An obvious difference is seen: the hot gas ejected by multiple $\mathrm{SNe}$ forms a "vortex" that acts to de-stabilize the swept-up shell of cold material via a Kelvin-Helmholtz instability. The shell loses its smooth elliptical form and develops "ripples" at its tops. The dynamical pressure of the hot gas (note that its velocity is much higher than in the case of a hypernova explosion) accelerates the "rippled" shell. As a consequence, the shell shows a strong Rayleigh-Taylor instability via the development of a characteristic "spike-and-bubble" morphology, as is seen in the left panel of Fig. 5. In the case of a hypernova explosion of the same total energy, the gas velocity field plotted in the right panel of Fig. 5 lacks any circular motion inside the shell. The smooth elliptical shell of cold material of roughly the same size as in the case of multiple SNe expands at roughly the sound speed. The shell has already started losing its pressure support (as implied by the 


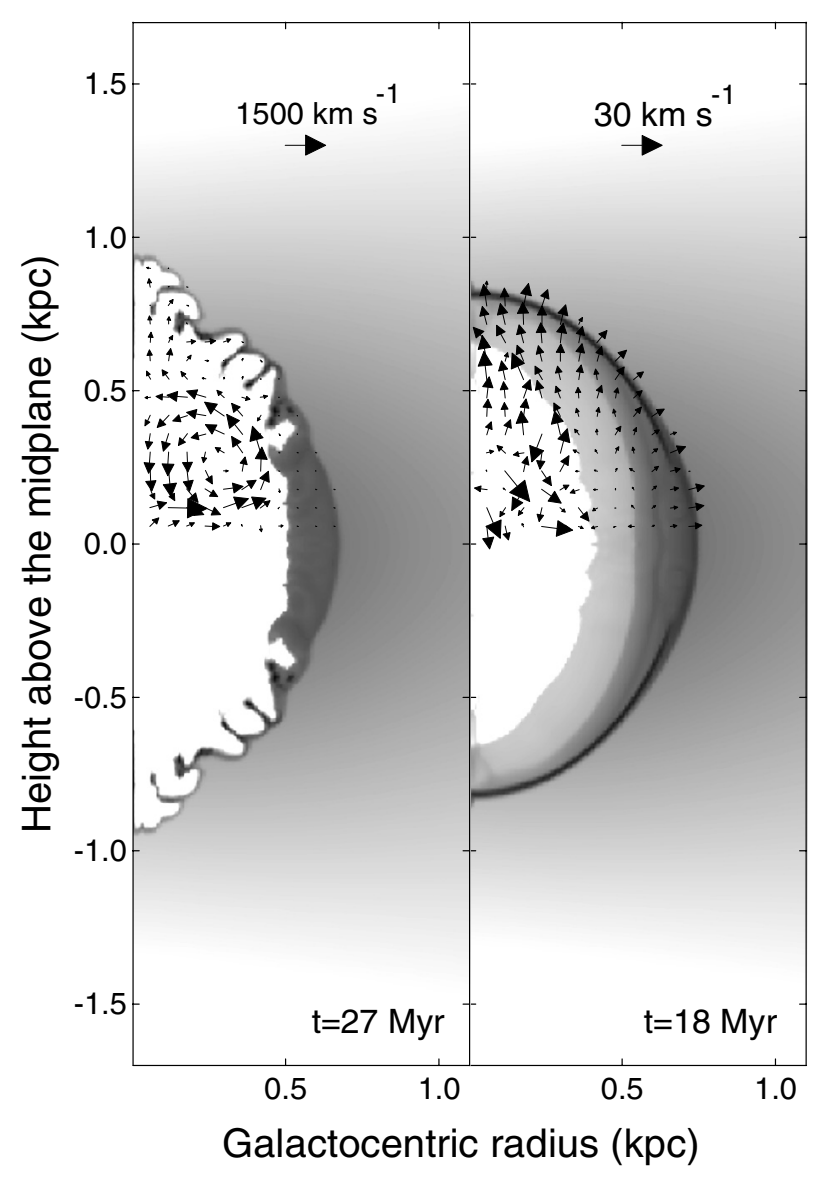

Fig. 5. The vertical cut through the distribution of the gas volume density produced by 500 consecutive $\mathrm{SNe}$ at $t=27 \mathrm{Myr}$ (the left panel) and single hypernova of the same total energy of $5.0 \times 10^{53} \mathrm{erg}$ (the right panel). The gas velocity field is shown by the arrows.

thickening of the shell walls) due to radiative cooling of its hot interior. As a consequence, the decelerating shell does not develop a Rayleigh-Taylor instability and the blowout phase is not observed. We note here that a hypernova-driven shell expands out to a certain radius faster than a shell created by multiple $\mathrm{SNe}$ with the same total energy, as reported by Efremov et al. (1999). Our numerical simulations have shown that the difference in the dynamics of shells produced by multiple SNe and a single hypernova of the same total energy intensifies as one considers more flattened gas systems. The maximum difference is found in the strongly vertically stratified gas distributions assumed for massive spiral galaxies.

The vortex forms since the shell becomes elongated in the vertical direction. Consider the simplified situation of hot gas ejected radially by $\mathrm{SNe}$ which is reflected from the cold dense walls of the elliptical shell. The gas will not be reflected along the local normal direction to the shell unless it is moving along $z=0$ or $r=0$. In general, a tangential component of the velocity of hot gas $v_{\mathrm{t}}$ (with respect to the shell's walls) is generated. It is always directed upwards for gas above the midplane and downwards for gas below the midplane. Let us consider the upper hemisphere. The occurrence of $v_{\mathrm{t}} \neq 0$ near the shell walls and the axial symmetry of the shell makes the hot gas (streaming upwards along the shell walls) accumulate at the top of the shell, because it cannot pass through the symmetry axis. Both the growing pressure of this hot gas (due to its negligible cooling) and the downward pull of the $z$-component of the galactic gravitational field lead to a downward flow along the axis of symmetry. This completes the circle and generates a vortex structure as seen in the left panel of Fig. 5. The development of such vortices is also seen in the axially symmetric numerical simulations of Recchi et al. (2001). However, a strong non-axisymmetry of the $\mathrm{SNe}$-driven shell may complicate the formation of the vortex.

The driving force for the vortex is a continuous or quasicontinuous release of energy by $\mathrm{SNe}$ of a stellar cluster or a group of closely located stellar clusters. This is also the reason why vortices do not form in a shell created by a single hypernova explosion. We have found that the vortex develops even when the number of $\mathrm{SNe}$ is quite moderate $(\sim 20)$ and the release of energy is discrete. The length scale of the vortex is approximately equal to the shell's semi-minor axis and time scale is limited by the duration of energy input from $\mathrm{SNe}$, i.e. $\$ 30$ Myr. In general, at least two vortices can co-exist within a single shell: one in the upper hemisphere and the other in the lower hemisphere of the shell.

Hypernovae tend to form complete shells. Such shells would appear in nearly face-on dwarf galaxies as HI rings with a low ring-to-center contrast in the HI column density. For instance, in Fig. 6 we plot the contrast in the HI column density between the ring and the central depression, $\mathcal{K}=N_{\mathrm{r}}(\mathrm{HI}) / N_{\mathrm{c}}(\mathrm{HI})$, as a function of the ring radius $R$ obtained for three different energy inputs. The ring column density $N_{\mathrm{r}}(\mathrm{HI})$ is computed by azimuthally averaging $N(\mathrm{HI})$ around the ring. An inclination angle (i.e. the angle between the rotation axis of the galaxy and the line of sight) of $i=5^{\circ}$ is assumed, which is appropriate for a nearly face-on galaxy. The solid lines give the contrast $\mathcal{K}$ for the multiple $\mathrm{SN}$ explosions, whereas the dashed lines do that for the hypernova explosion. As is seen, the ring-to-center contrast $\mathcal{K}$ produced by the hypernova explosion never exceeds 10 even for the upper limit energy of hypernova explosions of $10^{54} \mathrm{erg}$. On the other hand, multiple $\mathrm{SNe}$ can form $\mathrm{HI}$ rings with a much higher ring-tocenter contrast $\mathcal{K} \lesssim 100$. The latter takes place when the SNdriven shell is in the blowout phase. Note that the difference in $\mathcal{K}$ between the $\mathrm{HI}$ rings produced by multiple $\mathrm{SNe}$ and those produced by hypernovae smears out if the total released energy is $\leq 2 \times 10^{53} \mathrm{erg}$.

\subsection{PV-diagrams}

The position-velocity ( $\mathrm{pV}$ ) diagram is a powerful tool to search for coherent structures in the interstellar medium. In this section we compute model $\mathrm{pV}$-diagrams in order to determine their utility for distinguishing between the SN-driven shells and those produced by a hypernova explosion of the same total energy. The HI flux density $S_{\mathrm{HI}}$ is obtained using the following conversion formula (Binney \& Merrifield 1998):

$\frac{M_{\mathrm{HI}}}{\Delta v}=2.35 \times 10^{5}\left(\frac{D}{\mathrm{Mpc}}\right)^{2}\left(\frac{S_{\mathrm{HI}}}{\mathrm{Jy}}\right)\left[\frac{M_{\odot}}{\mathrm{km} \mathrm{s}^{-1}}\right]$, 


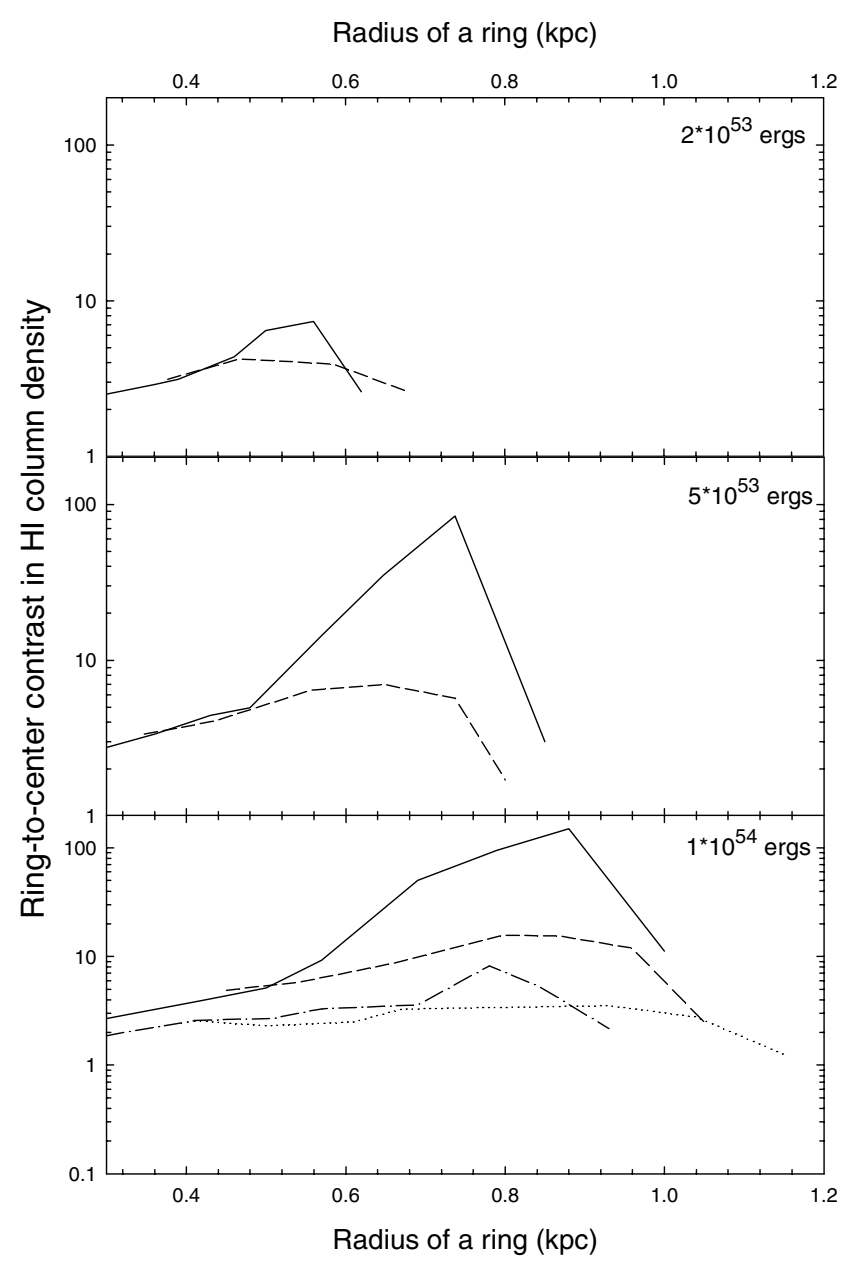

Fig. 6. The contrast in the HI column density between the ring and the central depression $\mathcal{K}=N_{\mathrm{r}}(\mathrm{HI}) / N_{\mathrm{c}}(\mathrm{HI})$ as a function of the ring radius. The solid lines show the contrast $\mathcal{K}$ produced by multiple $\mathrm{SNe}$, whereas the dashed lines show that produced by a single hypernova of the same total energy. An inclination angle of $5^{\circ}$ is assumed. The energy input is indicated in the right upper corner of each panel. The dashed-dotted and dotted lines in the lower panel give the contrast $\mathcal{K}$ for an inclination angle of $45^{\circ}$.

where for the distance $D$ we take $3.5 \mathrm{Mpc}$, appropriate for Holmberg I. The position-velocity cuts are taken along the major axis of the projected shell. The width of the cuts is $30 \mathrm{pc}$. We tried twice wider cuts $(60 \mathrm{pc})$, but found little difference in the appearance of the resulting $\mathrm{pV}$-diagrams as compared to those obtained with narrower cuts. The gas is assumed to be thermalized when constructing the model $\mathrm{pV}$-diagrams, i.e. a Maxwellian velocity distribution is assumed for the gas in each computational cell, with the scale determined by the local gas temperature (see Mashchenko \& Silich 1995). No attempt was made to include the effects of turbulence. The model galaxy is assumed to have an inclination angle of $5^{\circ}$. The positionvelocity cuts along the minor axis of the projected shell have similar appearance for such low inclination angles.

Figure 7 shows the model pV-diagrams of the shell produced by 500 consecutive SNe. The quantity $S_{\mathrm{HI}}$ is plotted in all model $\mathrm{pV}$-diagrams. Two bright blobs elongated in the vertical direction are apparent in each pV-diagram. They

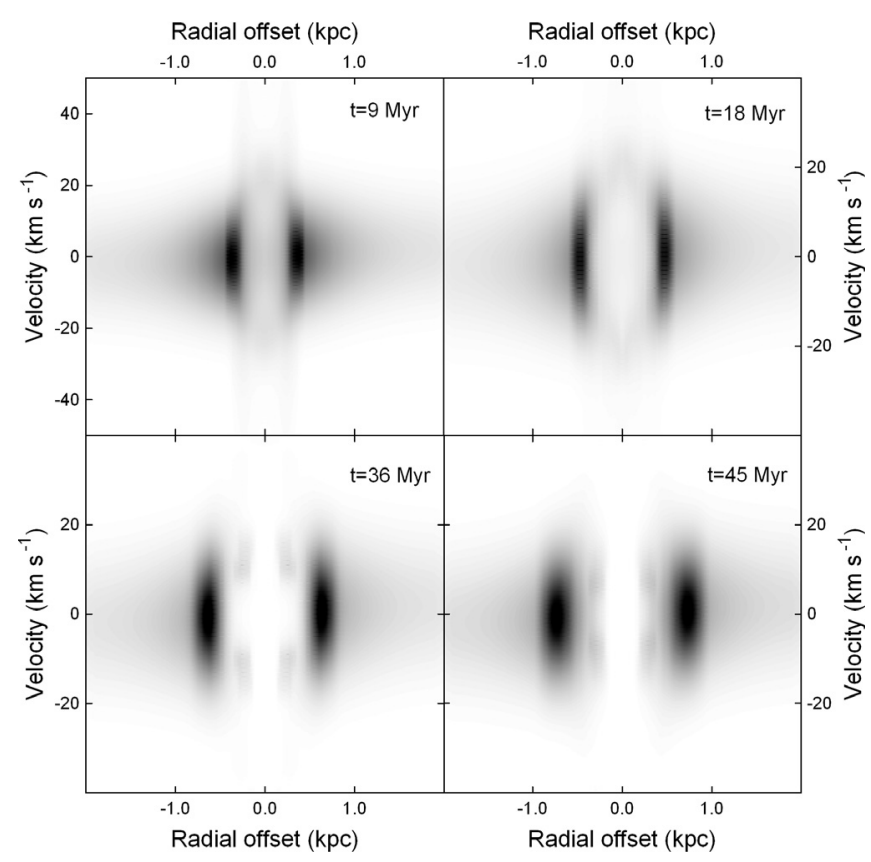

Fig. 7. The pV-diagrams of the shell created by 500 consecutive SNe. The $\mathrm{pV}$-cuts are taken along the major axis of the model galaxy viewed at an angle of $5^{\circ}$. The evolutionary time of the shell is indicated in the right upper corner. The quantity $S_{\mathrm{HI}}$ is plotted in all model pV-diagrams.

represent the dense walls of the shell expanding in the plane of the galaxy, i.e. perpendicular to the line of sight at the adopted inclination angle of $5^{\circ}$. If the shell has not yet broken out of the disk ( $t=9$ and $18 \mathrm{Myr}$ ), these two blobs appear to close at their tops and form a complete elliptical ring. The relative amplitude of $S_{\mathrm{HI}}$ around the ring is however varying by roughly an order of magnitude, reflecting the difference in the HI column density along the major axis of the projected shell - there is much less gas on the approaching and receding sides of the shell than on those expanding perpendicular to the line of sight.

The elliptical ring is virtually absent in the later expansion phase at $t=36$ and $45 \mathrm{Myr}$ when the shell has already broken out of the disk (the lower frames in Fig. 7). Instead, the $\mathrm{pV}$-diagrams are totally dominated by two high-intensity blobs. A few smaller patches seen between the blobs represent the dense, cold clouds fragmented off the shell and pushed by the dynamical pressure of the hot gas to a higher altitude of $\geq 1 \mathrm{kpc}$. The thickening of the shell after the blowout is also noticeable in the lower frames of Fig. 7. The same tendency is found in the $\mathrm{pV}$-diagrams of the shell created by 1000 consecutive SNe. The complete ring is seen only in the very early phase of the shell expansion at $t=9 \mathrm{Myr}$ and is virtually absent after the blowout at $t=27,45$, and $54 \mathrm{Myr}$.

Figure 8 shows the $\mathrm{pV}$-diagrams of the shell produced by a hypernova of total energy $5 \times 10^{53} \mathrm{erg}$. The position-velocity cuts are taken along the major axis of the projected shell at four different evolutionary times: $t=9,18,27$, and $36 \mathrm{Myr}$, i.e., before it has expanded out to its maximum size ( $t=9$ and $18 \mathrm{Myr})$ and after it has started to collapse due to the radiative cooling of the shell interior. An inclination angle $i=5^{\circ}$ is assumed. The elliptical hole surrounded by a ring of higher HI flux 


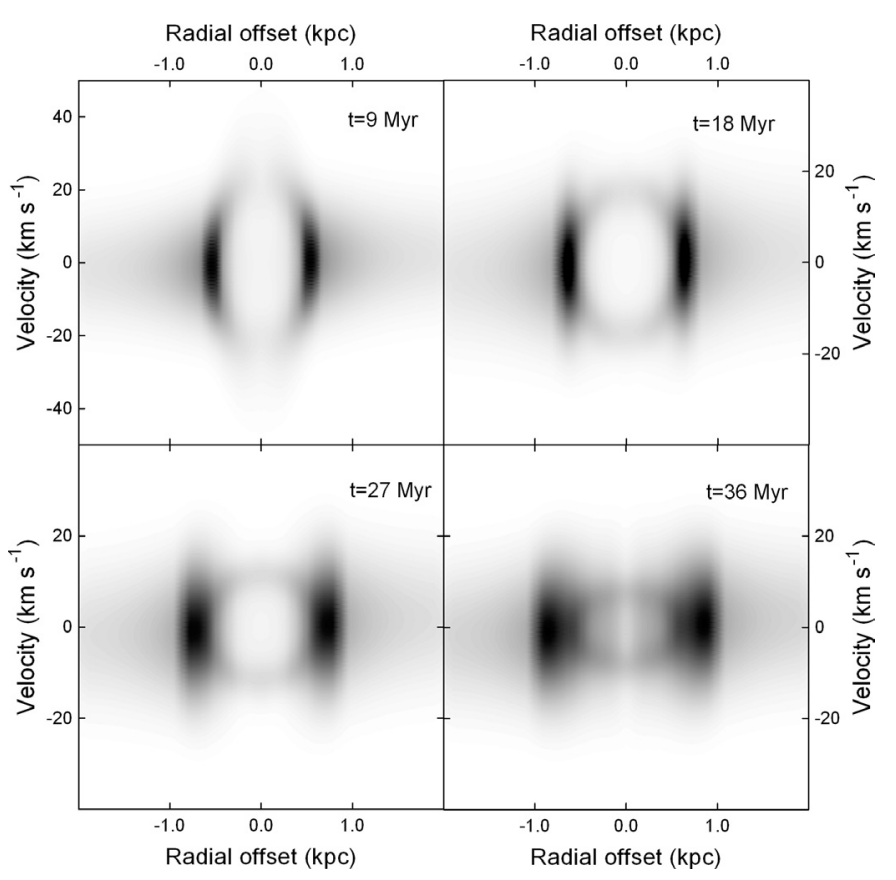

Fig. 8. The pV-diagrams of the shell produced by a single hypernova of the total energy of $5.0 \times 10^{53} \mathrm{erg}$. The $\mathrm{pV}$-cuts are taken along the major axis of the model galaxy viewed at an inclination angle of $5^{\circ}$.

density $S_{\mathrm{HI}}$ is clearly visible in those $\mathrm{pV}$-diagrams. The HI flux $S_{\mathrm{HI}}$ from the ring is $\sim 7-10 \mu \mathrm{Jy}$, which should, in principle, be detectable. Hypernova explosions tend to form complete shells that are clearly visible in the $p V$-diagrams. This characteristic feature of a hypernova-driven shell is also present when one considers very energetic explosions. For instance, Fig. 9 shows the $\mathrm{pV}$-diagrams of the shell produced by a hypernova of the total energy of $10^{54} \mathrm{erg}$. The elliptical hole surrounded by the ring is clearly seen even when the shell has already started to collapse at $t=45 \mathrm{Myr}$, as implied by the presence of a vertical bar at zero radial offset (see the lower right panel in Fig. 9). Note the size of the shell in Figs. 8 and 9. It is twice the local Gaussian scale height $h$, which is $\sim 350$ pc at a galactocentric radius of $0.5 \mathrm{kpc}$ in our model galaxy.

The comparison of Fig. 7-9 indicates that the SNe-driven shell in its late expansion phase (when its radius exceeds $1.5 h$ ) may be distinguished from that produced by the single hypernova explosion based on its appearance in the pV-diagrams. The $\mathrm{pV}$-diagrams of the hypernova-driven shell show the characteristic elliptical ring in virtually any evolutionary phase of the shell, whereas the $\mathrm{pV}$-diagrams of the $\mathrm{SN}$-driven shell do that only in the early expansion phase, well before the blowout. The present numerical simulations (see also Vorobyov \& Shchekinov 2004) show that the SN-driven shell breaks out of the disk when its radius exceeds $\sim 1.5 \mathrm{~h}$. As a consequence of the blowout, the $\mathrm{SN}$-driven shell transforms into an open cylinder and the $\mathrm{pV}$-diagrams show two parallel blobs representing the walls of the expanding cylindrical shell. On the contrary, the hypernova-driven shell appears to survive the expansion even when its radius exceeds $2.0 \mathrm{~h}$. As a result, the characteristic elliptical ring representing the complete expanding shell is clearly seen in the pV-diagrams. Thus, if the $\mathrm{pV}$-diagrams

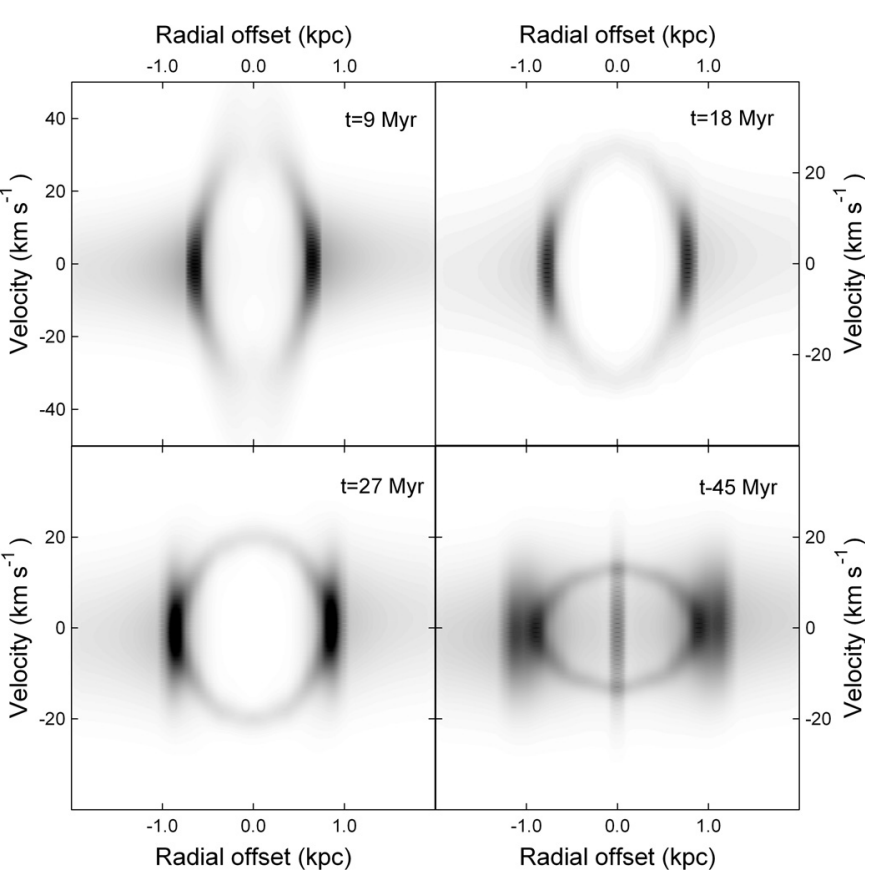

Fig. 9. The same as Fig. 8, but for a single hypernova of the total energy of $10^{54} \mathrm{erg}$.

show an elliptical hole surrounded by a denser ring, the radius of which is greater than $1.5 \mathrm{~h}$ (as in the lower frames of Figs. 8 and 9), this may indirectly point to a hypernova origin of the shell.

\subsection{Effects of inclination}

The model pV-diagrams considered in Sect. 4.1 were constructed under the assumption that the model galaxy is viewed nearly face-on, namely at an inclination angle of $5^{\circ}$. In this section we study how a higher inclination angle could influence the appearance of the SN- and hypernova-driven shells in the $\mathrm{pV}$-diagrams. Figure 10 shows the model pV-diagrams of the shell created by 1000 consecutive SNe. The pV-diagrams are constructed at three different evolutionary times $t=9,27$, and $45 \mathrm{Myr}$ after the beginning of the energy injection phase. The left and right panels in Fig. 10 are the position-velocity cuts along the minor and major axes of the projected shell, respectively. An inclination angle of $i=45^{\circ}$ is adopted. The characteristic hole surrounded by a ring of higher HI flux density (see Sect. 4.1) is barely seen even in the very early expansion phase at $t=9$ Myr due to the projection smearing at higher inclination angles. In the later expansion phase at $t=45 \mathrm{Myr}$ the $\mathrm{pV}$-cuts taken along the major axis show two elongated blobs, the morphological centers of which are symmetrically displaced by $|\Delta v| \sim 7-8 \mathrm{~km} \mathrm{~s}^{-1}$ with respect to $v=0 \mathrm{~km} \mathrm{~s}^{-1}$. The displacement is defined as $\Delta v= \pm v_{\text {rot }} \sin (i)$, where $v_{\text {rot }}$ is the rotation velocity of the $\mathrm{SN}$-driven shell. The positive and negative $\Delta v$ correspond to the receding and approaching parts of the shell, respectively. It is important to note here that similar pV-diagrams are obtained for Ho I by Ott et al. (2001, see their Fig. 8). A large displacement between the blobs with respect to the systemic velocity of $v_{\text {sys }}=141.5 \mathrm{~km} \mathrm{~s}^{-1}$ is clearly 


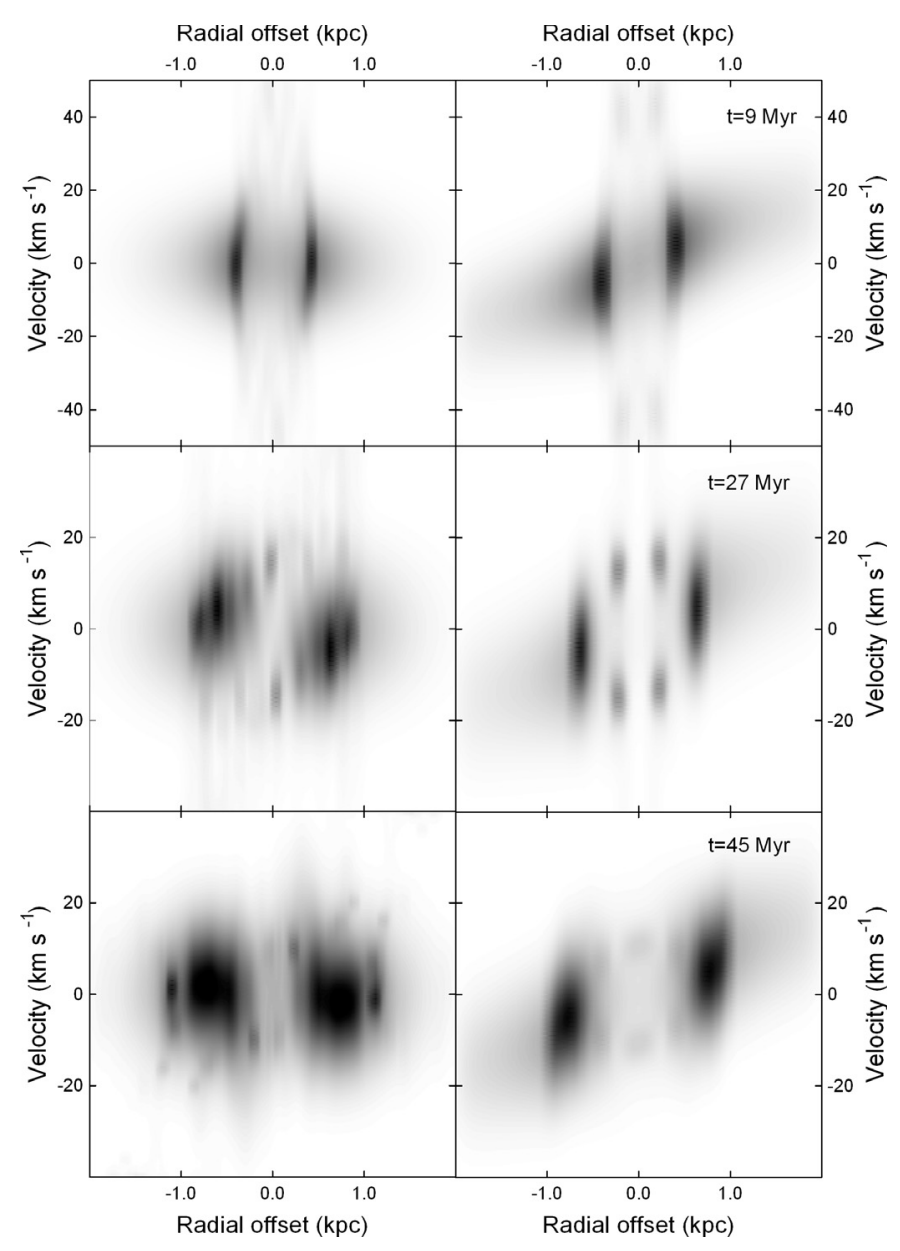

Fig. 10. The pV-diagrams of the shell created by 1000 consecutive $\mathrm{SNe}$. The pV-cuts are taken along the minor (the left panels) and the major (the right panels) axis of the model galaxy viewed at an angle of $45^{\circ}$.

seen in the $\mathrm{pV}$-cut taken along the major axis of Ho I. However, we found it difficult to estimate the value of $\Delta v$ from the observed pV-diagram shown in Fig. 8 of Ott et al. (2001) due to its considerable asymmetry (caused probably by an off-center location of the stellar cluster responsible for the creation of the shell). The modeled value of $\Delta v$ depends also on the adopted rotation curve of Ho I, which is poorly known (Ott et al. 2001). Therefore, it is problematic to obtain the inclination angle of Ho I by comparing the modeled and observed $\Delta v$. Nevertheless, we conclude that Ho I is viewed at an inclination angle considerably higher than $i=5^{\circ}$, because the modeled $\mathrm{pV}$-cuts of Fig. 7 obtained for an inclination angle of only $5^{\circ}$ do not show a noticeable displacement.

The $\mathrm{pV}$-diagrams of the shell created by a single hypernova explosion of the total energy $10^{54} \mathrm{erg}$ are shown in Fig. 11. The left panels give the $\mathrm{pV}$-cuts along the minor axis of the projected shell, whereas the right panels do those along the major axis. The pV-diagrams of the shell created by a single hypernova explosion are noticeably different from those created by multiple SNe. The hypernova-driven shell does not break out of the disk even when its radius exceeds $2.0 \mathrm{~h}$. As a consequence, the shell preserves its elliptical form and the characteristic elliptical hole surrounded by the ring of a higher HI

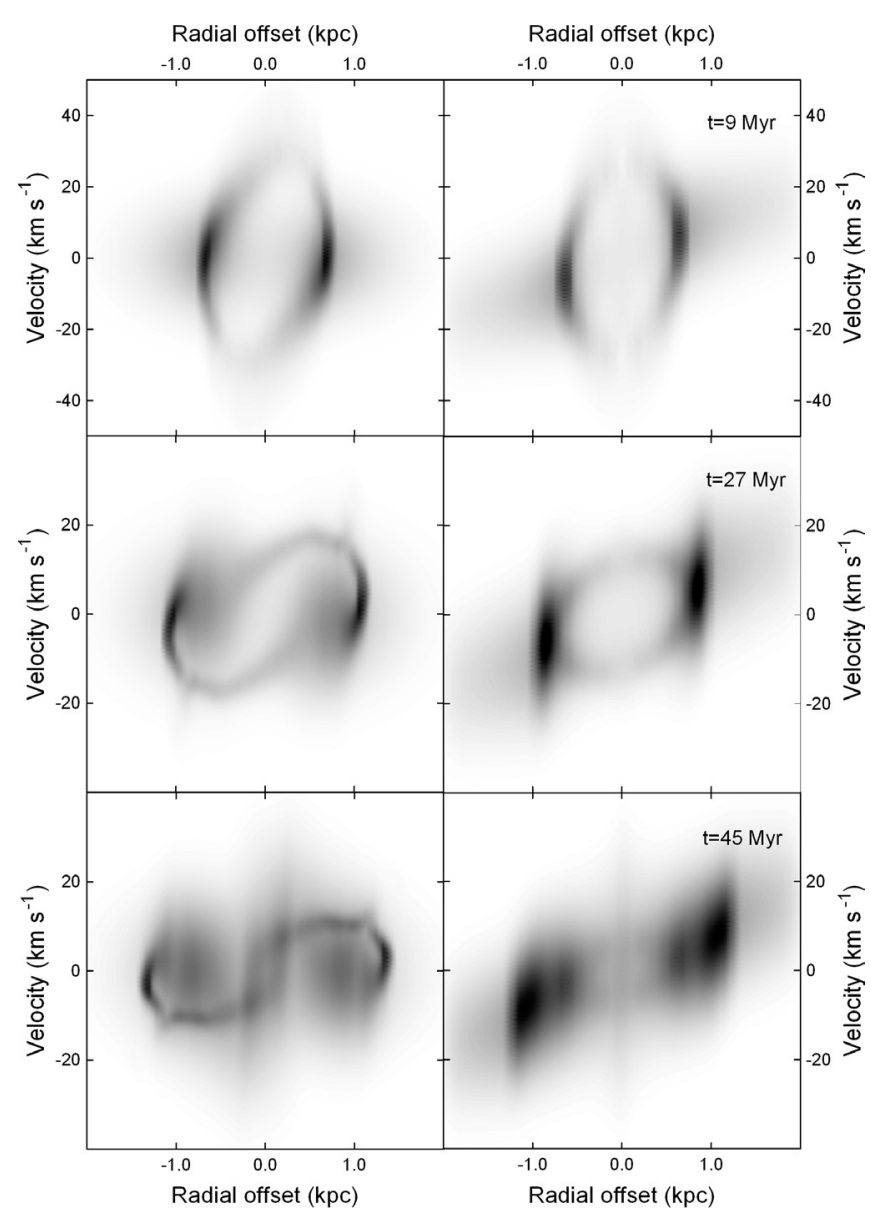

Fig. 11. The same as in Fig. 10, but for a single hypernova of the same total energy of $10^{54} \mathrm{erg}$. The $\mathrm{pV}$-cuts taken along the minor axis of the hypernova-driven shell show the characteristic S-shaped structure due to an expansion in the vertical direction.

flux intensity is apparent in the $\mathrm{pV}$-cuts taken along the major axis (the right panels in Fig. 11) at $t=9$ and $27 \mathrm{Myr}$ after the explosion. The elliptical ring dissolves at $t \geq 45 \mathrm{Myr}$ due to the projection effect (note that in nearly face-on galaxies the elliptical ring can still be resolved at that phase). In that later phase, the hypernova-driven shell can barely be distinguished from that created by multiple $\mathrm{SNe}$ based on the appearance in the $\mathrm{pV}$-diagrams taken along the major axis of the projected shell. Fortunately, the $\mathrm{pV}$-cuts taken along the minor axis of the hypernova-driven shell show the characteristic $\mathrm{S}$-shaped structure. The $\mathrm{pV}$-cuts along the minor axis are sensitive only to the expansion/contraction motion of the shell. As is seen in the lower panels of Fig. 4, the hypernova-driven shell at $t=27-45$ Myr expands in the vertical direction, but shows the lack of expansion or contraction motion in the galactic plane (except for the very late phase when the shell has already started to collapse). As a result, the shell seen in the $\mathrm{pV}$-diagrams as the elliptical ring transforms into the S-shaped structure in the later expansion phase. Note that neither elliptical rings nor $\mathrm{S}$-shaped structures are seen in the observed pV-diagrams of Ho I plotted in Fig. 8 of Ott et al. (2001).

As was demonstrated in Fig. 6, the HI rings created by multiple $\mathrm{SNe}$ have a much higher contrast in the HI column density 


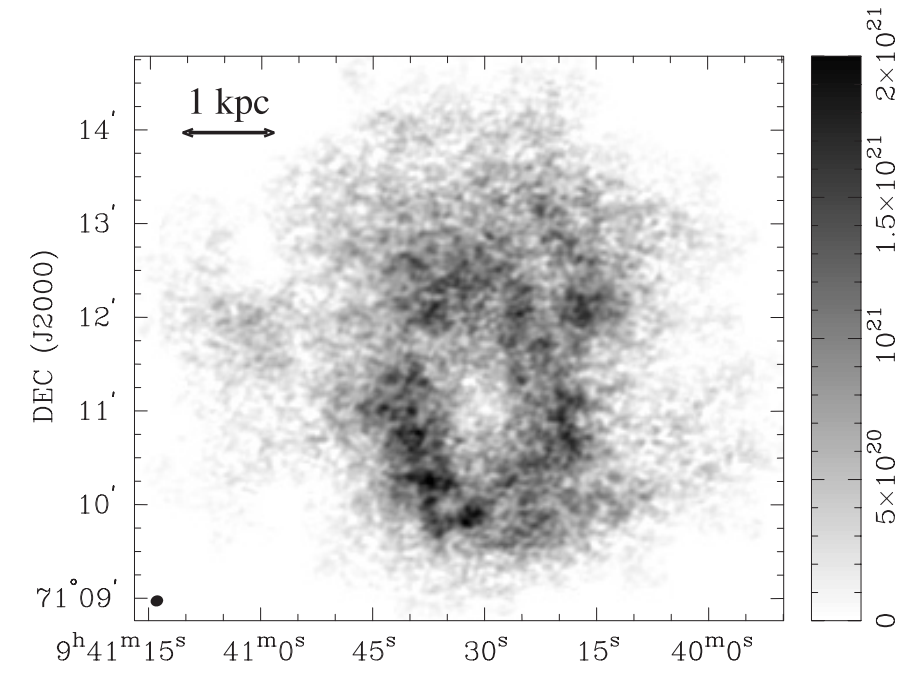

Fig. 12. Integrated HI emission of Ho I. In the bottom left corner we show the half-power beam $\left(8^{\prime \prime} .2 \times 7.0^{\prime}{ }^{\prime}\right)$. The grayscale represents the HI column density. The data have been obtained from the Very Large Array observations (Ott et al. 2001).

between the ring and central depression, $\mathcal{K}=N_{\mathrm{r}}(\mathrm{HI}) / N_{\mathrm{c}}(\mathrm{HI})$, than those created by a single hypernova explosion of the same total energy. However, a higher inclination may considerably reduce the value of $\mathcal{K}$ due to the projection smearing effect. For instance, the dotted-dashed line in Fig. 6 shows the contrast $\mathcal{K}$ as a function of the ring radius $R$ produced by 1000 consecutive $\mathrm{SNe}$, whereas the dotted line does that for the hypernova of the same total energy. An inclination angle of $45^{\circ}$ is assumed. The ring-to-center contrast in $N(\mathrm{HI})$ produced by both multiple $\mathrm{SNe}$ and hypernova stays around $\mathcal{K}=2-3$. The only exception is seen when the $\mathrm{SN}$-driven shell expands out to $R \approx 0.7 \mathrm{kpc}$ and breaks out of the disk. Then, the ring-to-center contrast reaches a maximum value of $\mathcal{K} \approx 8$. It is instructive to compare the ring-to-center contrast and size of $\mathrm{HI}$ rings produced in our simulations (by both multiple SNe and single hypernovae) with the observed contrast $\mathcal{K}$ and size of the HI ring in Ho I. Figure 12 shows the observed HI map of Ho I as obtained by Ott et al. (2001). The diameter of the ring is $\sim 1.7 \mathrm{kpc}$ and the ring-to-center contrast is $\mathcal{K} \sim 15-20$. Our simulations show (see Fig. 6) that both multiple SNe and single hypernovae may form rings of up to $2 \mathrm{kpc}$ in diameter. However, single hypernovae fail to produce rings with the observed contrast of $\mathcal{K} \sim 15-20$. This is true even in the most favorable case of near zero inclination angle. Taking into account that both the observations of Ott et al. and numerical modeling of Vorobyov et al. (2004) set the lower limit on the inclination angle of Ho I as $i \leq 15^{\circ}$, the formation of the HI ring with such a high contrast $\mathcal{K} \sim 15-20$ by means of a hypernova explosion seems highly problematic. Apparently, only multiple $\mathrm{SNe}$ can produce $\mathrm{HI}$ rings with such a high ring-to-center contrast $\mathcal{K}$.

The integrated HI image of the shell created by multiple $\mathrm{SNe}$ appears in nearly face-on galaxies as a ring of elevated HI density surrounding the central depression. For instance, the left upper panel in Fig. 13 shows the integrated $\mathrm{HI}$ image of the shell created by 500 consecutive SNe. The HI image is taken at $t=45 \mathrm{Myr}$ after the beginning of the energy input phase, when

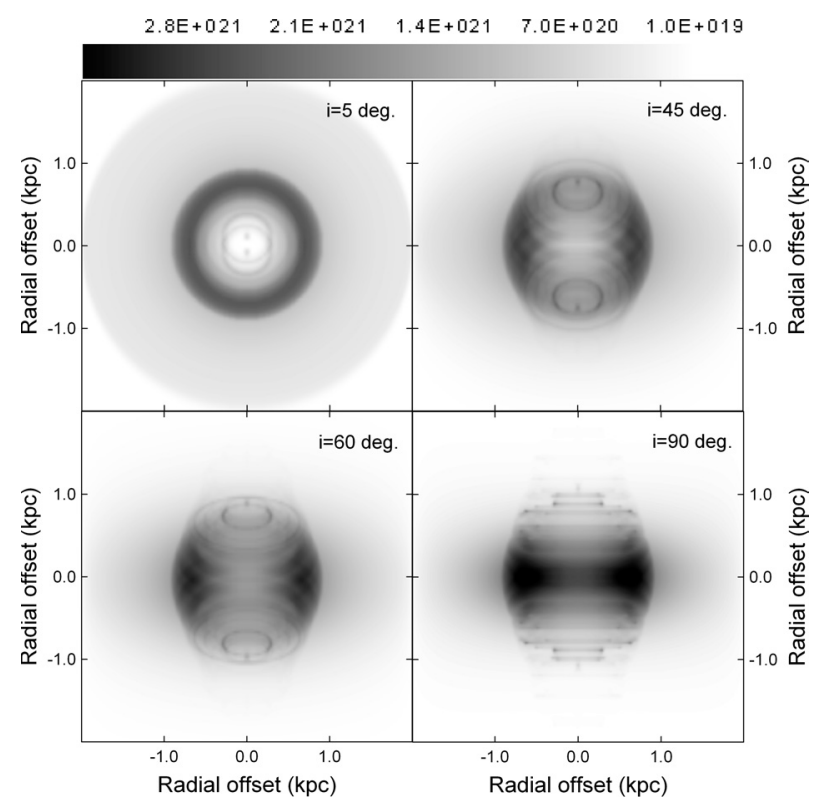

Fig. 13. The integrated HI image of the ring produced by 500 consecutive SNe. The inclination angle is indicated in the right upper corner. The scale bar is in $\mathrm{cm}^{-2}$. The origin of narrow rings is an assumption of axisymmetry in our numerical simulations.

the shell has already broken out of the disk. An inclination angle of $i=5^{\circ}$ is assumed. As is obviously seen, the blown-out shell in nearly face-on galaxies appears as a ring with a high ring-to-center HI column density contrast ( 25-30). There is little azimuthal variation in the HI column density around the ring. However, the integrated $\mathrm{HI}$ image of the blownout shell observed in substantially inclined galaxies shows a considerable variation in the HI column density around the ring. For instance, at higher inclination angles of $45^{\circ}$ and $60^{\circ}$ the relative amplitude of azimuthal variations in $N(\mathrm{HI})$ around the ring is $\approx 2$ and $\approx 3$, respectively. In this case, the integrated HI image is dominated by two kidney-shaped density enhancements and shows a mild central minimum. It is interesting to note that the Sextans A and M 81 dwA dwarf irregular galaxies have a somewhat similar HI distribution (see Skillman et al. 1988; Stewart 1998). In the case of Sextans A, the relative amplitude of azimuthal variations in the HI column density around the ring is $\approx 2.5-3$ (according to Skillman et al. 1988) and the inclination angle determined from the outer HI contours is $i=36^{\circ} \pm 4^{\circ}$ (assuming the circular symmetry). Note that the narrow ring-shaped structures seen in Fig. 13 are in fact the dense fragments of a broken-up shell, which appear as rings rather than dense clumps due to the axisymmetric nature of our numerical simulations. In edge-on galaxies, the integrated HI image would resemble a "dumbbell", as shown in the right lower panel of Fig. 13. The absence of noticeable azimuthal variations in HI column density around the HI ring in Fig. 12 suggests that Ho I is viewed at an inclination angle considerably smaller than $i=45^{\circ}$. This is in agreement with previous estimates by Ott et al. (2001) and Vorobyov et al. (2004), who found that Ho I has an inclination angle of $\lesssim 20^{\circ}$. 


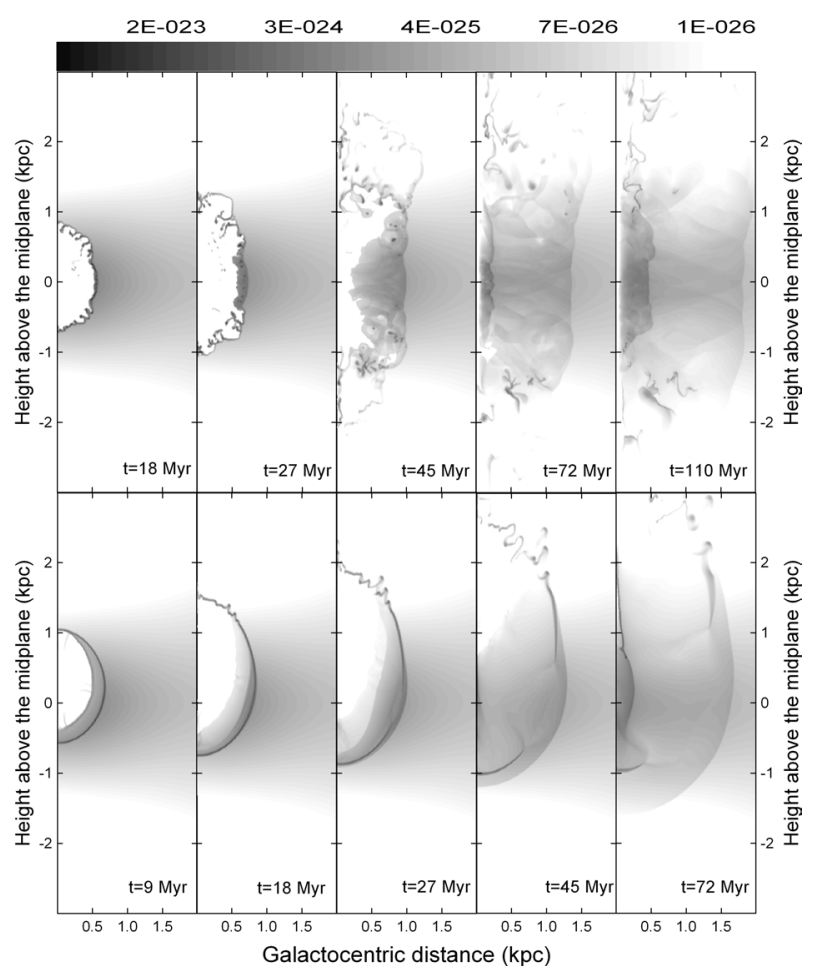

Fig. 14. Off-plane explosions. Temporal evolution of the gas volume density distribution after the release of $1.0 \times 10^{54} \mathrm{erg}$ of thermal energy at $r=0, z=100 \mathrm{pc}$. The upper panels correspond to 1000 consecutive $\mathrm{SN}$ explosions over $30 \mathrm{Myr}$, while the lower panels show the impact of a single hypernova of the same total energy of $10^{54} \mathrm{erg}$. The scale bar is in $\mathrm{gm} \mathrm{cm}^{-3}$.

\subsection{Off-plane explosions}

A general assumption made in the above simulations of both SN-driven and hypernova-driven shells was that the energy is released exactly in the midplane of the model gas disk. However, taking into account the considerable thickness of the gas disks in dIrr's, the occurrence of a hypernova or massive stellar cluster at a position moderately offset with respect to the galactic midplane, appears plausible. In this section we investigate how the off-plane explosions may influence the appearance of the shells in the pV-diagrams.

If the stellar cluster is located at a moderate height of $z=100$ pc $(\sim 1 / 3 h)$ above the midplane of the galaxy, the temporal evolution of the gas volume density is found to be similar to that obtained in Sect. 4 for the midplane stellar cluster. For instance, the upper panels in Fig. 14 show five temporal snapshots of the gas volume density distribution created by the energy release of 1000 consecutive SNe. A more intensive blowout takes place in the upper part of the galaxy where the stellar cluster is nested. However, the $\mathrm{pV}$-diagrams and ringto-center contrast in HI column density $\mathcal{K}$ are only slightly affected by this mild asymmetry.

The temporal evolution of the shell created by a single hypernova that is moderately offset with respect to the midplane of the galaxy produces some noticeable differences. The lower panels in Fig. 14 give five temporal snapshots of the gas volume density distribution created by the hypernova of total energy $10^{54}$ erg that is placed $100 \mathrm{pc}$ above the midplane of the galactic gas disk. The egg-like shell is formed after $\approx 15 \mathrm{Myr}$, the upper part of which retains a relatively smooth appearance for several tens of Myr before it breaks due to a RayleighTaylor instability and dissolves at $\approx 70-80$ Myr after the explosion. Note that in the case of multiple SNe such smooth kpc-sized arcs of dense material are never formed in our numerical simulations. Instead, inspection of Figs. 2-4, and 14 indicates that multiple $\mathrm{SNe}$ tend to produce patchy filaments and dense clumps of a few hundred parsecs in size (except for the very late phase when the shell has already collapsed). Indeed, a Rayleigh-Taylor instability, the effect of which is strengthened by the preceding Kelvin-Helmholtz instability, efficiently fragments the SN-driven shell when the latter breaks out of the gas disk. Longer fragmentation times of hypernova-driven shells as compared to fragmentation times of shells created by multiple $\mathrm{SNe}$ were also found in the model of Efremov et al. (1999). It is interesting to mention in this context the W4 HII region in the Perseus arm of the outer Galaxy. Dennison et al. (1997) have observed a highly elongated shell of $\mathrm{H} \alpha$ emission, the base of which is located near the W4 HII region. The $\mathrm{H} \alpha$ shell appears to close far above the Galactic plane and is interpreted by Basu et al. (1999) to be the dense ionized wall of swept-up gas surrounding a bubble of hot rarefied gas created by the stellar winds from the massive stars. However, the apparent smooth appearance of the $\mathrm{H} \alpha$ shell implies that the Rayleigh-Taylor instability is suppressed there, possibly due to the stabilizing effect of a swept-up tangential magnetic field in the shell (Komljenovic et al. 1999). An alternative explanation may involve a shell formed by a hypernova explosion. In that case, the characteristic growth time of a Rayleigh-Taylor instability in the expanding shell is much longer than in the case of multiple SNe, and the shell may survive for a longer time and expand to a higher altitude.

Off-plane hypernova explosions may leave noticeable signatures in the $\mathrm{pV}$-diagrams. While the upper part of the egg-like shell is thin and contains a small fraction of the total gas mass, the lower part of the shell accumulates a larger amount of gas mass and becomes clearly visible in the $\mathrm{pV}$-diagrams. Figure 15 shows the pV-cuts taken along the major axis of the shell created by a single hypernova of the total energy of $10^{54} \mathrm{erg}$, which is placed $100 \mathrm{pc}$ above the midplane of the galaxy. An inclination angle of $5^{\circ}$ is adopted. The bottom of the egg-like shell appears in the $\mathrm{pV}$-diagrams as a bright arc connecting two vertical blobs. The latter are the walls of the shell expanding in the horizontal direction. The top of the shell is clearly visible only in the very early expansion phase at $t \leq 10 \mathrm{Myr}$. However, it could be seen in $\mathrm{H} \alpha$ emission, if a powerful ionizing source is located inside the shell, as might be the case for the W4 HII region discussed above.

\section{High velocity clouds}

In this section we numerically study the vertical impact of high velocity clouds as a potential mechanism of the giant HI ring formation in Ho I. The numerical simulations of multiple $\mathrm{SNe}$ in Sect. 4 have shown that it requires $>2.0 \times 10^{53}$ erg of released energy to form $\mathrm{HI}$ rings with $\mathrm{HI}$ ring-to-center contrast $\mathcal{K}>10$. Hence, we consider only very energetic HVCs 


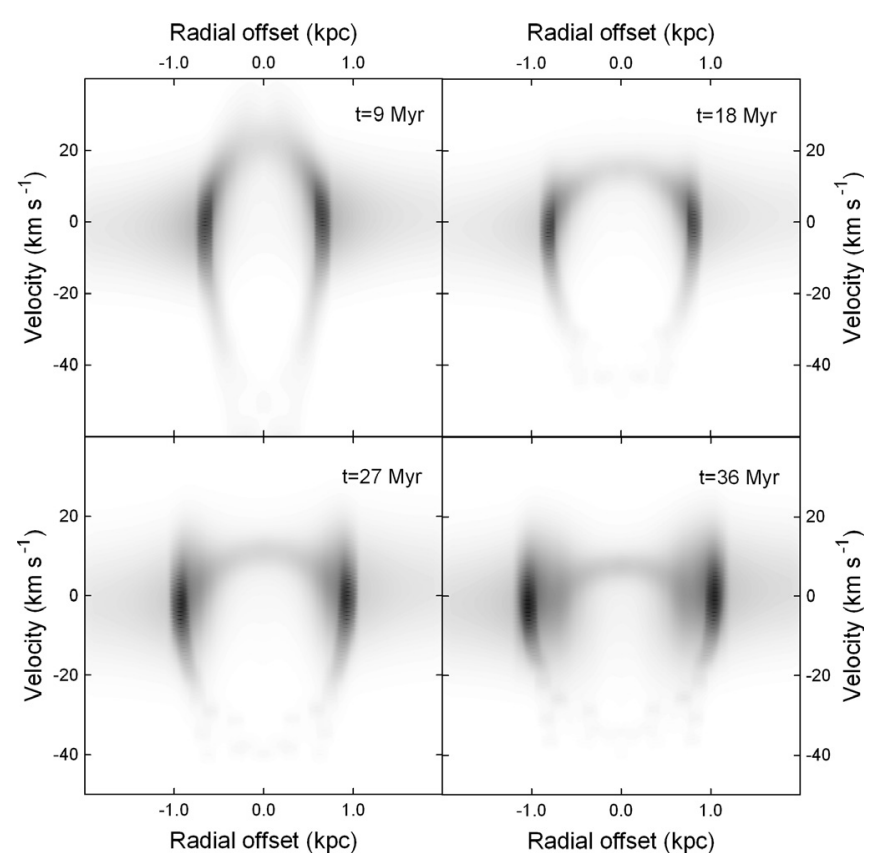

Fig. 15. Off-plane explosion. The $p V$-diagrams of the shell created by a single hypernova of the total energy of $10^{54} \mathrm{erg}$. The $\mathrm{pV}$-cuts are taken along the major axis of the model galaxy viewed at an inclination angle of $5^{\circ}$.

that could potentially deposit a comparable amount of kinetic energy to the interstellar medium. We have performed a parameter study assuming that HVCs cover a range of velocities $200 \mathrm{~km} \mathrm{~s}^{-1} \leq v_{\mathrm{HVC}} \leq 300 \mathrm{~km} \mathrm{~s}^{-1}$ and masses $2.5 \times 10^{5} M_{\odot} \leq$ $M_{\mathrm{HVC}} \leq 2.5 \times 10^{6} M_{\odot}$. Thus, the kinetic energies of HVCs range from $1.0 \times 10^{53}$ to $2.5 \times 10^{54} \mathrm{erg}$. Clouds are assumed to have a constant density and be either spherical or cylindrical in shape. The initial position of the cloud center is $3.2 \mathrm{kpc}$ above the midplane.

Figure 16 shows the temporal evolution of the distribution of the gas volume density produced by the impact of a cloud of $M_{\mathrm{HVC}}=2.5 \times 10^{6} M_{\odot}$ and $v_{\mathrm{HVC}}=200 \mathrm{~km} \mathrm{~s}^{-1}$. The total kinetic energy of the cloud is thus equal to $1.0 \times 10^{54} \mathrm{erg}$. The maximum column density $N(\mathrm{HI})$ of the cloud measured along its diameter is $4.3 \times 10^{20} \mathrm{~cm}^{-2}$. The impinging HVC creates a hot rarified bubble by pushing matter toward the galactic plane. The bubble does not form a complete sphere and fills out $\sim 40 \mathrm{Myr}$ after the onset of the collision. The HVC continues to push the gas along its way and eventually breaks through the disk. At this stage $(t \approx 110 \mathrm{Myr})$, the initial shape of the HVC is completely lost due to a combined action of Rayleigh-Taylor and thermal instabilities. The HVC emerges above the galactic plane as a complicated network of dense filaments, clumps, and arcs. The formation of dense filaments and clumps was also reported in numerical simulations by Franco et al. (1988). It takes another $70 \mathrm{Myr}$ for this remnant to fall back onto the disk and create another bubble, though less prominent than the first one.

Although the kinetic energy of the impinging cloud is high $\left(10^{54} \mathrm{erg}\right)$ and the cloud produces a considerable disturbance in the gas disk of the target galaxy, we have found that it is rather ineffective in creating giant $\mathrm{HI}$ rings similar to that observed

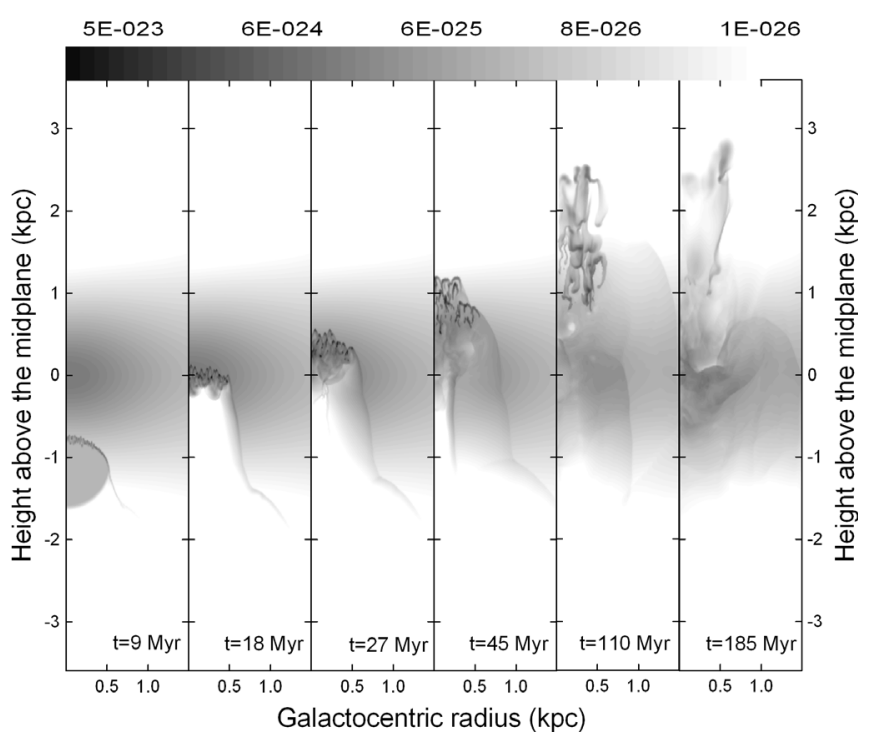

Fig. 16. The distribution of the gas volume density created by the collision of a cloud of $M_{\mathrm{HVC}}=2.5 \times 10^{6} M_{\odot}$ and $v_{\mathrm{HVC}}=200 \mathrm{~km} \mathrm{~s}^{-1}$ with the galactic gas disk.

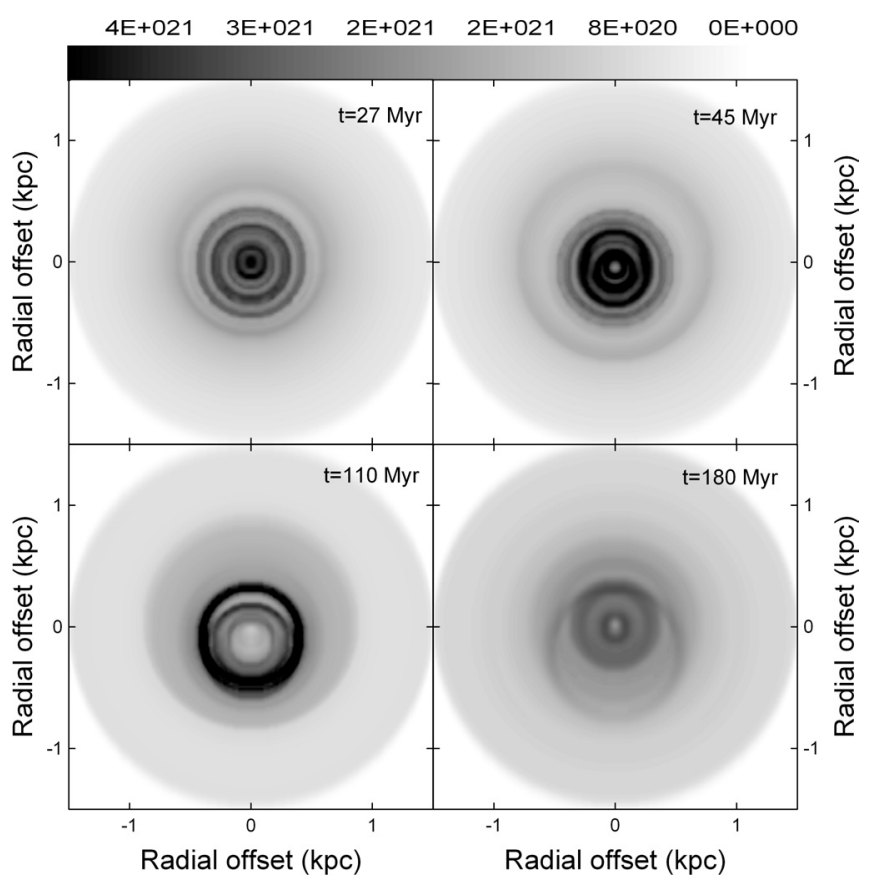

Fig. 17. The integrated HI image of the gas volume density distribution shown in Fig. 16. An inclination angle of $5^{\circ}$ is adopted. The scale bar is in $\mathrm{cm}^{-2}$.

in Ho I. The HVC pushes the matter mostly in the vertical direction so that the $\mathrm{HI}$ image of a nearly face-on galaxy does not show a considerable radial variation in the HI column density within the impact region. Indeed, Fig. 17 shows the integrated $\mathrm{HI}$ image of the gas volume density distribution shown in Fig. 16. An inclination angle of $5^{\circ}$ is assumed. The formation of a ring-like structure is only seen in the late collision phase ( $t \sim 110 \mathrm{Myr}$ ), when the cloud has plunged through the gas disk and pushed a considerable fraction of the disk gas to an altitude $z \gtrsim 1 \mathrm{kpc}$. Most of the gas constituting this remnant retains the orbital momentum it had before the collision. 
Since the horizontal gravity is much less at $z \gtrsim 1 \mathrm{kpc}$ than in the plane of the galaxy, the lifted gas starts spiraling away on ballistic orbits and it can be observed in nearly face-on galaxies as a dense HI ring surrounding the central depression (see the left lower panel in Fig. 17). The maximum size of such an $\mathrm{HI}$ ring (diameter $\sim 0.8 \mathrm{kpc}$ ) is at least twice smaller than that found in our numerical simulations of multiple SNe and hypernova explosions of the same total energy. Note that the diameter of the HI ring in Ho I is $\sim 1.7 \mathrm{kpc}$. We find that most of the HI rings formed by the impact of HVCs have diameters less than $0.5 \mathrm{kpc}$. The HVCs of a smaller size and higher HI column density, but the same kinetic energy, tend to form smaller rings. The size of the HI ring depends weakly on the shape of the cloud. HVCs with the total kinetic energy below $2 \times 10^{53}$ do not form noticeable $\mathrm{HI}$ rings. HI rings with a diameter of $\geq 0.8 \mathrm{kpc}$ are never formed in our simulations for the considered upper limit of HVC kinetic energy, $2.5 \times 10^{54} \mathrm{erg}$.

A more dramatic difference between the HI rings formed by the vertical impact of HVCs and those created by multiple $\mathrm{SNe}$ is seen in the values of $\mathcal{K}$. As is obviously seen from Fig. 6 , 1000 consecutive $\mathrm{SNe}$ can create rings with $\mathcal{K}$ as high as 100 , whereas Fig. 17 indicates that the impact of a cloud of the same kinetic energy generates the ring with $\mathcal{K} \approx 4.5$. Note that $\mathcal{K}$ in Holmberg I is $\sim 15-20$ (Ott et al. 2001). The same tendency is found for other values of released energy: multiple SNe produce rings with roughly 20-30 times higher values of $\mathcal{K}$ than does the impact of a cloud of the same total energy. On the other hand, hypernova explosions form rings with $\mathcal{K}$ roughly a factor of 2 higher than those created by the clouds of the same kinetic energy. The difference in $\mathcal{K}$ between the rings formed by the three mechanisms considered above is smeared out as one considers higher inclination angles of $i>45^{\circ}$.

The impact of HVCs should leave prominent features in the pV-diagrams, which could be used to distinguish between the rings formed by the impact of HVCs and those formed by multiple SN and hypernova explosions. Indeed, Fig. 18 shows the $\mathrm{pV}$-cuts taken along the major axis of the galaxy experiencing the collision with a cloud of $M_{\mathrm{HVC}}=2.5 \times 10^{6} M_{\odot}$ and $v_{\mathrm{HVC}}=200 \mathrm{~km} \mathrm{~s}^{-1}$ An inclination angle of $5^{\circ}$ is adopted. The open bubble produced by the impinging cloud is clearly seen in the upper left panel of Fig. 18. The bubble starts filling out after $t=45$ Myr. However, the cloud is still clearly visible in the $\mathrm{pV}$-diagrams at that time as a dense irregular clump moving towards the observer. The velocity of the cloud noticeably decreases during the passage from $v_{\mathrm{HVC}} \approx 200 \mathrm{~km} \mathrm{~s}^{-1}$ at the onset of collision to $v_{\mathrm{HVC}} \approx 20 \mathrm{~km} \mathrm{~s}^{-1}$ at the time when the cloud emerges on the other side of the gas disk. The $\mathrm{pV}$ diagram at this time $(t=110 \mathrm{Myr})$ is dominated by two bright and compact clumps, which represent the cold dense gas compressed and pushed by the cloud to an altitude of $z \approx 1-2 \mathrm{kpc}$. The depression in the HI flux seen in the $\mathrm{pV}$-diagram near the galactic center is due to the HI ring formation discussed above. Note that these two clumps are noticeably different in shape from those observed in the $\mathrm{pV}$-diagrams of the shells formed by multiple SN and hypernova explosions (see Figs. 7-9). The second bubble formed by the remnant falling back onto the disk is clearly seen in the lower right panel of Fig. 18.

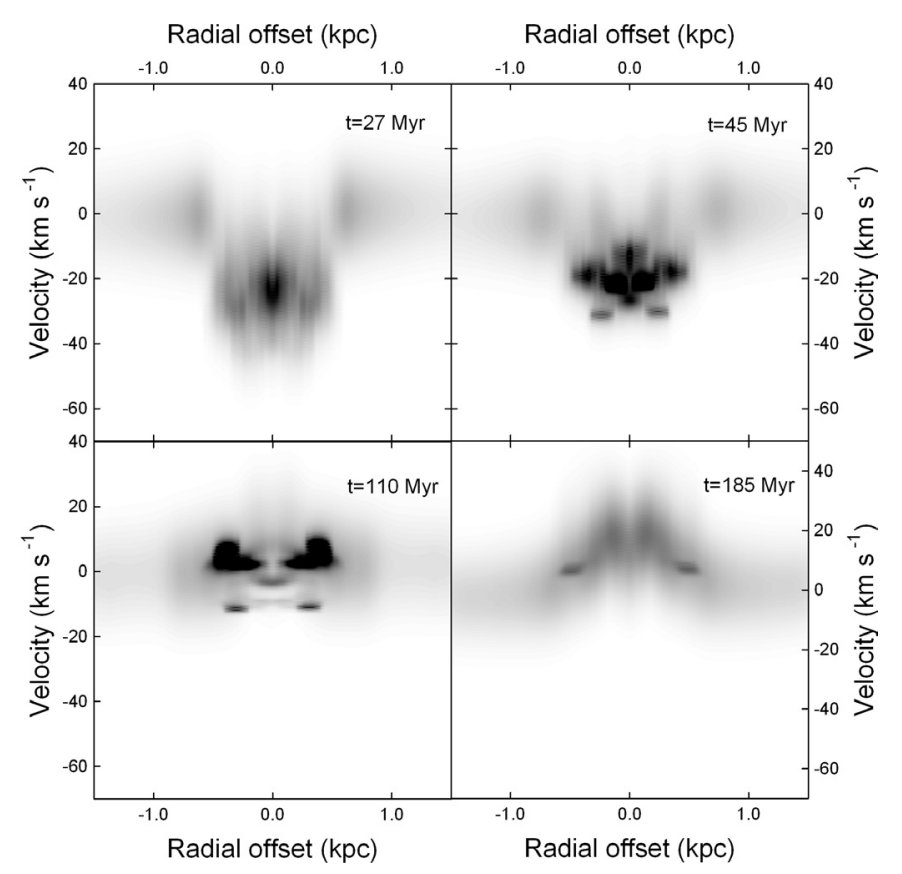

Fig. 18. The pV-diagrams of the gas volume density distribution shown in Fig. 16. The pV-cuts are taken along the major axis of the projected galaxy. An inclination angle of $5^{\circ}$ is assumed.

It is obviously seen that neither the $\mathrm{pV}$-diagrams nor the size and/or ring-to-center contrast $\mathcal{K}$ obtained in the simulations of the vertical collision of HVCs with the galactic disk can account for what is actually observed in Ho I. We thus conclude that multiple SN explosions remain the most plausible explanation for the origin of the giant HI ring morphology in Ho I.

\section{Discussion}

If $\mathrm{SNe}$ are the origin of $\mathrm{HI}$ rings surrounding $\mathrm{HI}$ holes, the presence of a remnant stellar cluster is expected. Such stellar clusters are not always observed within HI rings or holes. For instance, Rhode et al. (1999) found that several HI holes in the dwarf irregular galaxy Holmberg II lack any stellar cluster counterparts. However, in the case of Holmberg I, the $B$ band magnitude as well as the $U-B$ color of the optical emission from the giant HI hole indicates that a stellar cluster of $\sim 2 \times 10^{5} M_{\odot}$ may be present there (Vorobyov et al. 2004).

The high density clumps produced in our simulations due to the fragmentation of SNe-driven shells are usually not selfgravitating. The masses of these clumps are slightly below the Jeans mass $M_{\mathrm{J}}=5 c_{\mathrm{s}}^{2} d /(4 G)$, where $d$ is the size of the clump. This expression is derived from the virial theorem assuming spherical clumps. This is consistent with a low $\mathrm{H} \alpha$ luminosity of Ho I: $L_{\mathrm{H} \alpha}=4.3 \times 10^{38} \mathrm{erg} \mathrm{s}^{-1}$ (Miller \& Hodge 1996). This also implies that $\mathrm{SNe}$ alone have difficulty in producing self-gravitating clumps in dwarf irregular galaxies, and probably an external agent such as ram pressure or the collision of two or more SNe-driven shells is needed to induce star formation. In massive disk galaxies, radiative cooling is usually more efficient than in dwarf irregulars due to a higher metallicity. Therefore, dense clumps are more likely to become Jeans 
unstable. On the other hand, the most energetic HVC's with kinetic energy $\gtrsim 1.0 \times 10^{54} \mathrm{erg}$ can indeed produce dense clumps that are Jeans unstable. However, such energies are rather exceptional and hence this mechanism cannot account for most star formation in dwarf irregulars.

There are a few assumptions inherent to the model that need further discussion.

The interstellar medium distribution in Ho I. The solution of the steady state momentum equation in Sect. 2 produces a smooth initial gas distribution, which may be only a first order approximation to the more realistic inhomogeneous interstellar medium of dwarf irregulars. The influence of the deviations from a smooth ISM distribution in the gas disk on the formation and evolution of HI shells deserves a separate detailed study. We expect here that the effect of small scale inhomogeneities with sizes much smaller than that of the HI shell should be cancelled out as the shell forms. Conversely, large scale inhomogeneities with sizes comparable to that of the HI shell could influence its shape and evolution, making it easier/harder to break out of the disk. In particular, large scale inhomogeneities may reduce the smooth appearance of hypernova-driven shells discussed in Sect. 4.3. However, considering the size of the HI ring in Ho I ( $\sim 1.7 \mathrm{kpc})$, we do not expect inhomogeneities of such a scale to be present in the initial gas distribution of this galaxy.

The vertical scale height of the stellar disk and gas velocity dispersion. The actual value of the vertical scale height $z_{\mathrm{s}}$ of the stellar disk is difficult to estimate, since Ho I has a low inclination angle. We have adopted a value of $z_{\mathrm{s}}=300 \mathrm{pc}$, which is typical for dwarf irregular galaxies. A moderate variation $\pm 100 \mathrm{pc}$ in the value of $z_{\mathrm{s}}$ has a minor influence on the gas distribution and, hence, on our main results. The HI velocity dispersion of Ho I is measured by Ott et al. (2001, see their Fig. 10). A moderate value $\sigma_{\mathrm{HI}} \approx 9 \mathrm{~km} \mathrm{~s}^{-1}$ is found for most of Ho I disk, which is typical for gas-rich, quiet dwarf galaxies (Stil 1999) and is used in our numerical simulations. A small portion of gas disk in the northwestern part of Ho I has, however, a slightly higher value of $\sigma_{\mathrm{HI}} \approx 12 \mathrm{~km} \mathrm{~s}^{-1}$. Such a variation in $\sigma_{\mathrm{HI}}$ is not expected to influence our main results. On the other hand, the measured $\sigma_{\mathrm{HI}}$ represents the cold component of the gas disk only. If a massive hot gas component is present in Ho I, the velocity dispersion $\sigma_{\mathrm{HI}+\mathrm{HII}}$ of the mixture of hot and cold gas components may be significantly greater. An increased gas velocity dispersion makes the gas disk thicker. For instance, for an adopted value of $\sigma_{\mathrm{HI}+\mathrm{HII}}=20 \mathrm{~km} \mathrm{~s}^{-1}$, the Gaussian scale height of the model gas disk $h$ becomes roughly three times larger than that shown by the dotted line in Fig. 1. However, $h \gtrsim 1 \mathrm{kpc}$ is not expected in Ho I, because a SN-driven shell with radius $r \approx 0.7-0.8 \mathrm{kpc}$ would not blow out of the disk. Therefore, we would not observe such a high column density contrast $(\mathcal{K} \approx 15-20$ ) between the HI ring and the central depression. Furthermore, Ho I is a quiet dwarf galaxy with an average star formation rate of only $0.004 M_{\odot} \mathrm{yr}^{-1}$ (Miller \& Hodge 1996) and we do not expect a large amount of hot gas to be present there. Hence, we conclude that the integrated gas velocity dispersion of Ho I is not expected to deviate much from the adopted value of $\sigma=9 \mathrm{~km} \mathrm{~s}^{-1}$.

Internal structure and geometry of HVCs. The shape of HVCs (i.e. spherical or cylindrical) is found to have a very minor influence on our results, which is in agreement with many previous studies of HVC collisions with a gas disk (e.g. Comeron \& Torra 1992). The assumption of an initially homogeneous cloud is certainly an idealized case, since the observations show that HVCs are very clumpy and hierarchically structured (e.g. Wakker \& Schwarz 1991). It may be that the remnant of the initially homogeneous cloud that emerges at the other side of the galactic plane at $t \approx 110 \mathrm{Myr}$ in Fig. 16 is a better example of an HVC prior to the collision. Inhomogeneous structure further reduces the ability of an HVC to produce large HI rings.

\section{Conclusions}

We have numerically studied the formation of a giant HI ring as is observed in the Holmberg I dwarf irregular galaxy (Ott et al. 2001). The following three energetic mechanisms with the total released energy of $\$ 10^{54}$ erg have been considered: multiple $\mathrm{SNe}$, a hypernova explosion associated with a gamma ray burst, and the vertical impact of an HVC. The shells created by those mechanisms are seen in the integrated HI image of a nearly face-on galaxy as the HI rings of various sizes and ring-to-center contrasts $\mathcal{K}$ in the HI column density. We find the following differences among the HI rings formed by those mechanisms:

- Multiple SNe form rings with the highest ring-to-center contrast $\mathcal{K} \lesssim 100$. A single hypernova and an HVC form rings with much smaller values of $\mathcal{K} \lesssim 10$ and $\mathcal{K} \lesssim 5$, respectively. This is due to the difference in the dynamics of the shells produced by multiple $\mathrm{SNe}$ and those created by a hypernova: multiple $\mathrm{SNe}$ are much more effective in producing blowout due to a combined action of Kelvin-Helmholtz and Rayleigh-Taylor instabilities than are single hypernovae of the same total energy. The vertical impact of an HVC is found to be ineffective in evacuating the gas in the radial direction and hence in creating HI rings with a high ring-tocenter contrast. It is important to note that the difference in $\mathcal{K}$ found among the three energetic mechanisms smears out at an inclination angle $i \geq 45^{\circ}$ due to the projection effect.

- Both multiple SNe and a hypernova can account for the observed size of the HI ring in Ho I (diameter $\sim 1.7 \mathrm{kpc}$ ). The maximum size of the ring that an $\mathrm{HVC}$ is found to form in our numerical simulations is $\sim 0.8 \mathrm{kpc}$. We thus conclude that only multiple SNe can reproduce both the size (diameter $\sim 1.7 \mathrm{kpc}$ ) and the ring-to-center contrast in the HI column density $(\mathcal{K} \sim 15-20)$ of the HI ring in Ho I.

We construct the model position-velocity diagrams and find that they can be used to distinguish between HI rings produced by different energetic mechanisms. The model $\mathrm{pV}$-diagrams of the blownout shell produced by multiple SNe are similar to those observed in Ho I and show two symmetrically displaced, elongated blobs representing the walls of the shell. On the other hand, the model pV-diagrams of the shell created by a hypernova explosion show a characteristic elliptical ring-like structure, which is not seen in the observed pV-diagrams of Ho I. The impact of HVCs leaves prominent features in the model pV-diagrams that are also not found in the observed 
pV-diagrams of Ho I. Those features include dense irregular clumps and a void representing the impinging cloud and the bubble formed by the impact. We thus conclude that multiple SNe appear to be the most plausible energetic mechanism of the HI ring formation in Ho I.

Our numerical simulations indicate that the appearance of the SN-driven shell in dwarf irregulars may depend on the inclination of the galaxy. In nearly face-on galaxies the blownout shell appears in the integrated HI image as an HI ring with a deep central minimum. There is little azimuthal variation in the HI column density around the ring. The integrated HI image of the blownout shell in considerably inclined galaxies $\left(i \geq 45^{\circ}\right)$ is instead dominated by two kidney-shaped enhancements and shows a mild central minimum, which is similar to what is found in Sectans A and M 81 dwA (Skillman et al. 1988; Stewart 1998). In nearly edge-on galaxies the integrated HI image of the blownout shell resembles a "dumbbell".

The offplane explosion of a hypernova creates the egg-like shell that can preserve its shape for a considerably longer time than the shell created by multiple $\mathrm{SNe}$ of the same total energy. This may explain the smooth appearance of the highly elongated $\mathrm{H} \alpha$ shell observed by Dennison et al. (1997) in the W4 HII region of the Perseus arm.

Acknowledgements. The authors are thankful to the referee, Prof. J. Palouš, for valuable suggestions and comments that helped improve the manuscript. This work was supported by the NATO Science Fellowship Program administered by the Natural Sciences and Engineering Research Council (NSERC) of Canada. E.V. is grateful to the staff of the Department of Physics and Astronomy, University of Western Ontario for their hospitality.

\section{References}

Basu, S., Johnstone, D., \& Martin, P. G. 1999, ApJ, 516, 843

Binney, J., \& Merrifield, M. 1998, in Galactic Astronomy, ed. P. Ostriker, \& D. N. Spergel (Princeton University Press), 442

Binney, J., \& Tremaine, S. 1987, Galactic Dynamics (Princeton: Princeton Univ. Press)

Brinks, E. 1990, in The Interstellar Medium of Galaxies, ed. H. Thronson, \& J. Shull (Dordrecht: Kluwer), 39

Comerón, F., \& Torra, J. 1992, A\&A, 261, 94

Dennison, B., Topasna, G. A., \& Simonetti, J. H. 1997, ApJ, 474, L31

De Young, D., \& Heckman, T. M. 1994, ApJ, 431, 598

Efremov, Yu. N., Elmegreen, B. G., \& Hodge, P. W. 1998, ApJ, 501, L163
Efremov, Yu. N., Ehlerová, S., \& Palouš, J. 1999, A\&A, 350, 457

Elmegreen, B. G. 1997, ApJ, 477, 196

Franco, J., Tenorio-Tagle, G., Bodenheimer, P., Rózyczka, \& Mirabel, I. F. 1988, ApJ, 333, 826

Komljenovic, P. T., Basu, S., \& Johnstone, D. 1999, in New Perspectives on the Interstellar Medium, ed. A. R. Taylor, T. L. Landecker, \& G. Joncas (San Francisco: ASP), ASP Conf. Ser., 168, 299

Loeb, A., \& Perna, R. 1998, ApJ, 503, L35

Mac Low, M.-M., \& Ferrara, A. 1999, ApJ, 513, 142

Mac Low, M.-M., \& McCray, R. 1988, ApJ, 324, 776

Mashchenko, S. Ya., \& Silich, S. A. 1995, AZh, 72, 660

Miller, B. W., \& Hodge, P. 1996, ApJ, 458, 467

Ott, J., Walter, F., Brinks, E., et al. 2001, AJ, 122, 3070

Paczyński, B. 1998, ApJ, 494, 45

Persic, M., Salucci, P., \& Stel, F. 1996, MNRAS, 281, 27

Puche, D., Westpfahl, D., Brinks, E., \& Roy, J.-R. 1992, AJ, 103, 1841

Rand, R. J., \& Stone, J. M. 1996, AJ, 111, 190

Rhode, K. L., Saltzer, J. J., Westpfahl, D. J., \& Radice, L. A. 1999, AJ, 118, 323

Recchi, S., Matteucci, F., \& D'Ercole, A. 2001, MNRAS, 322, 800

Sánchez-Salcedo, F. J. 2002, Rev. Mex. Astron. Astrofís., 38, 39

Sargent, E. L. W., Sancisi, R., \& Lo, K. Y. 1983, ApJ, 265, 711

Silich, S., \& Tenorio-Tagle, G. 2001, ApJ, 552, 91

Stone, J. M., \& Norman, M. L. 1992, ApJS, 80, 753

Young, L. M., \& Lo, K. Y. 1997, ApJ, 490, 710

Skillman, E. D., Terlevich, R., Teuben, P. J., \& van Woerden, H. 1988, A\&A, 198, 33

Stewart, S. 1998, Ph.D. Thesis, The University of Alabama

Stil, J. A. 1999, Ph.D. Thesis, Univ. Leiden

Taylor, C., Brinks, E., Pogge, R. W., \& Skillman, E. D. 1994, AJ, 107, 971

Tenorio-Tagle, G., Franco, J., Bodenheimer, P., \& Rózyczka, M. 1987, A\&A, 179, 219

Tully, R. B., Bottinelli, L., Fisher, J. R., et al. 1978, A\&A, 63, 37

Vorobyov, E. I., Klein, U., Shchekinov, Yu. A., \& Ott, J. 2004, A\&A, 413, 939

Vorobyov, E. I., \& Shchekinov, Yu. A. 2004, A\&A, 416, 499

Wada, K., \& Norman, C. 2001, ApJ, 547, 172

Wada, K., Spaans, M., \& Kim, S. 2000, ApJ, 540, 797

Wakker, B. P., \& Schwarz, U. J. 1991, A\&A, 250, 484

Walter, F., \& Brinks, E. 1999, AJ, 118, 273

Walter, F., \& Brinks, E. 2001, AJ, 121, 3026

Weaver, R., McCray, R., Castor, J., Shapiro, P., \& Moore, R. 1977, ApJ, 218, 377

Westpfahl, D., \& Puche, D. 1993, in ESO Conf. Workshop Proc. 49, Dwarf Galaxies, ed. G. Meylan, \& P. Prugniel (Garching: ESO), 295 